\title{
Interplay of Spin and Spatial Anisotropy in Low-Dimensional Quantum Magnets with Spin 1/2
}

\author{
Alžbeta Orendáčová *, Róbert Tarasenko, Vladimír Tkáč, Erik Čižmár, Martin Orendáč and \\ Alexander Feher ${ }^{D}$
}

Institute of Physics, Faculty of Science, P.J. Šafárik University, Park Angelinum 9, 04154 Košice, Slovakia; robert.tarasenko@upjs.sk (R.T.); tkac.vladimir@upjs.sk (T.V.); erik.cizmar@upjs.sk (E.Č.); martin.orendac@upjs.sk (M.O.); alexander.feher@upjs.sk (A.F.)

* Correspondence: alzbeta.orendacova@upjs.sk; Tel.: +421552342280

Received: 26 November 2018; Accepted: 19 December 2018; Published: 21 December 2018

\begin{abstract}
Quantum Heisenberg chain and square lattices are important paradigms of a low-dimensional magnetism. Their ground states are determined by the strength of quantum fluctuations. Correspondingly, the ground state of a rectangular lattice interpolates between the spin liquid and the ordered collinear Néel state with the partially reduced order parameter. The diversity of additional exchange interactions offers variety of quantum models derived from the aforementioned paradigms. Besides the spatial anisotropy of the exchange coupling, controlling the lattice dimensionality and ground-state properties, the spin anisotropy (intrinsic or induced by the magnetic field) represents another important effect disturbing a rotational symmetry of the spin system. The $S=1 / 2$ easy-axis and easy-plane XXZ models on the square lattice even for extremely weak spin anisotropies undergo Heisenberg-Ising and Heisenberg-XY crossovers, respectively, acting as precursors to the onset of the finite-temperature phase transitions within the two-dimensional Ising universality class (for the easy axis anisotropy) and a topological Berezinskii-Kosterlitz-Thouless phase transition (for the easy-plane anisotropy). Experimental realizations of the $S=1 / 2$ two-dimensional XXZ models in bulk quantum magnets appeared only recently. Partial solutions of the problems associated with their experimental identifications are discussed and some possibilities of future investigations in quantum magnets on the square and rectangular lattice are outlined.
\end{abstract}

Keywords: Heisenberg; $S=1 / 2$ XXZ model; spin anisotropy; square lattice; chain; rectangular lattice; Berezinskii-Kosterlitz-Thouless phase transition; phase diagram; quantum magnet

\section{Introduction}

The history of low-dimensional magnetism started in 1925 by the theoretical work of Ising who found an exact solution of the hypothetic system of spins arranged into a chain and oriented in one direction [1]. Famous Onsager's solution of the two-dimensional Ising model on a square lattice [2] showed the existence of long-range order (appearance of nonzero spontaneous magnetization) at a finite temperature, $T_{\mathrm{C}}$. Intensive theoretical studies of one-dimensional (1D) and two-dimensional (2D) spin systems were stimulated by the effort to understand the properties of three-dimensional (3D) phase transitions and critical phenomena [3].

In 1966, Mermin and Wagner theoretically proved the absence of conventional long-range order (LRO) at a finite temperature in 1D and 2D Heisenberg (isotropic) and easy-plane (XY) magnets [4]. Nevertheless, Stanley and Kaplan conjectured a possibility of an unusual phase transition for 2D Heisenberg ferromagnet on triangular, square and honeycomb lattice arguing that the absence of a spontaneous magnetization at finite temperatures does not imply the absence of any phase transition [5]. Subsequent work indicated that the evidence for this was stronger for XY than for 
Heisenberg models [6-8]. Berezinskii revealed that eigenstates of the Hamiltonian describing the 2D lattice of planar rotators, 2D Bose liquid, and 2D XY magnet can be sorted into two classes; localized "vortices" characterized by a nonzero circulation along a minimum closed contour of the square lattice and displaced harmonic oscillations-spin waves with zero circulation $[9,10]$. Below a critical temperature related to some phase transition, the vortices form configurations with a total zero circulation. Kosterlitz and Thouless introduced a definition of a topological long-range order adopted from the dislocation theory of melting [11]. In a 2D crystal, authors showed that at low temperatures, dislocations with Burgers vector of the magnitude $b$ tend to form closely bound dipole pairs with resulting $b=0$. Above some critical temperature, the pairs start to dissociate and the dislocations will appear spontaneously. The same type of argument can be applied for the 2D XY model and 2D neutral superfluid. While in the 2D XY model, a logarithmically large energy barrier, $V(r) \sim-\ln (r)$, stabilizes a topological order formed by the bound pairs of vortices, in the case of the 2D Heisenberg model, there is no topological order, since energy barriers separating different configurations are small, allowing continuous changes between individual configurations [11].

Many theoretical studies showed that phase transitions in the 2D systems such as granular superconducting films, superfluid films, 2D Coulomb gas etc., with continuous symmetry of the order parameter, may be described by the 2D XY model [12]. These topological phase transitions are related to the dissociation of the pairs of topological excitations (vortices in superconducting or superfluid films, dislocations in 2D crystals, dipole pairs of oppositely charged particles in 2D plasma, etc.), and belong to the same universality class. In later literature, these transitions were named as Berezinskii-Kosterlitz-Thouless (BKT) transition, occurring at a finite critical temperature, $T_{\text {BKT }}$.

Unlike the theory, the move of low-dimensional magnets from the abstract to real world started much later, during the period of seventies and eighties, when the first real materials appeared, resembling the behavior theoretically predicted for the 1D and 2D magnetic models [13].

The physics of low-dimensional systems is interesting in its own right and some phenomena have no parallel in three-dimensional physics. Besides the absence of the aforementioned conventional $\mathrm{LRO}$ at finite temperatures, the main feature of low-dimensional systems is a failure of the classical spin-wave theory which is not able to describe the complexity of the non-linear spin dynamics [14]. It was realized that localized solitary excitations, large-amplitude waves propagating with a permanent profile, are possible in classical magnetic chains [15]. The classical theory of solitons has been remarkably successful in describing the properties of real magnetic chains as $\left(\mathrm{CH}_{3}\right)_{4} \mathrm{NMnCl}_{3}(\mathrm{TMMC})$ with spin $S=5 / 2$ and even $\left(\mathrm{C}_{6} \mathrm{H}_{11} \mathrm{NH}_{3}\right) \mathrm{CuBr}_{3}$ (CHAB) with $S=1 / 2$ [16]. Various theoretical approaches tried to find a quantum analog to the classical solitary excitations leading to the concept of quantum solitons which proved useful for the description of the ground-state properties of an $S=1$ Heisenberg antiferromagnetic (HAF) chain in the famous Haldane's conjecture $[17,18]$. Within the semi-classical approximation Haldane showed that the ground state of the HAF chain with integer spin is characterized by the presence of topological solitons. Consequently, the ground state is disordered with $S=0$, separated from the excited $S=1$ magnon state by the Haldane gap, arising from the presence of strong quantum fluctuations preventing the onset of the Néel order even at $T=0$. Experimentally, the Haldane phase was most comprehensively studied in the $S=1$ chain material $\mathrm{Ni}\left(\mathrm{C}_{2} \mathrm{H}_{8} \mathrm{~N}_{2}\right)_{2} \mathrm{NO}_{2} \mathrm{ClO}_{4}$ (NENP), confirming the theoretical predictions $[19,20]$.

The presence of strong magnetic fluctuations in the low-dimensional magnetic subsystems of high- $T_{C}$ superconductors triggered renewed intensive theoretical and experimental interest in the 2D quantum magnets [21,22]. In this context, the frustration effects became widely studied to understand the pairing processes. Sophisticated mathematical and computational methods enabled theorists to solve the variety of more complex low-dimensional quantum frustrated lattices including Shustry-Shuterland [23], Kagomé lattice [24], Kitaev honeycomb model [25] and others, having exotic properties and many of them still waiting for their discovery in the real world [26].

Besides the study of rather exotic 1D and 2D magnetic models, the theorists try to incorporate the effect of inter-chain/inter-layer coupling, crystal field, spin anisotropy, dilution and other 
effects present in real compounds. While a close collaboration of chemists, experimental and theoretical physicists working in the area of low-dimensional magnetism has long-lasting tradition, the participation of quantum chemists in this field appeared only recently. The community realized the large importance of quantum-mechanical calculations based on the first principles which can become crucial for the identification of the studied material. This cooperation is also stimulated by the practical needs-the search for variety of materials involving aforementioned low-dimensional and strongly frustrated systems which can be potentially used in nanotechnologies [27], quantum computing [28-30], refrigerating due to enhanced magneto-caloric effect [31-33], etc. Besides the aforementioned practical applications and solutions of fundamental problems, current low-dimensional magnetism is also characterized by the search for the analogies in different fields of physics (quantum tunneling [34], Bose-Einstein condensation [35], quantum phase transitions [36,37], thermal Hall effect [38], etc.).

This brief introduction to the history and current state of the low-dimensional magnetism tried to point out that this field covers extremely wide area of research comprising directions which seem to run independently until they mix together forming new qualities. In this context, this modern, widely developing area changes to a field with a strong interdisciplinary character based on the close interaction of theory and experiment, accompanied by the intensive collaboration of physicists, chemists and material engineers.

In this review, we will restrict to the ground-state and finite-temperature properties of the $S=1 / 2$ models interpolating between the quantum Heisenberg antiferromagnetic chain and the square lattice. Both models represent important paradigms of the low-dimensional magnetism. Their ground states are completely different, since they are determined by different strength of quantum fluctuations. The involving of additional exchange interactions results in the variety of the quantum models which can be derived from the aforementioned paradigms. Besides the spatial anisotropy of the exchange coupling which controls the lattice dimensionality, the spin anisotropy (intrinsic or induced by magnetic field) plays an important role in the symmetry of the order parameter. Despite the fact, that some of the models including the square lattice were theoretically studied many years ago, their experimental realizations appeared only recently, mostly in the bulk $\mathrm{Cu}(\mathrm{II})$ based metal-organic magnets. The problems associated with their experimental identifications are also discussed.

\section{Spatial Anisotropy of the Exchange Coupling: From the Chain to the Square Lattice}

\subsection{The $S=1 / 2$ Heisenberg Antiferromagnetic Chain}

Let us consider a pair of the $S=1 / 2$ spins coupled by the isotropic Heisenberg interaction

$$
H=J S_{1} \cdot S_{2}
$$

where $J>0$ corresponds to an antiferromagnetic (AF) interaction. The ground state of the coupled spins is represented by the singlet state

$$
|s\rangle=\frac{1}{\sqrt{2}}(|\uparrow \downarrow\rangle-|\downarrow \uparrow\rangle)
$$

with the energy $-3 J / 4$. The quantum-mechanical state represented by the Equation (2) is known as a valence bond and can be regarded as the ultimate expression of quantum fluctuations which govern the formation of more exotic magnetic states depending on the lattice symmetry, spin value etc.

$$
H=J \sum_{i}\left(S_{i}^{x} S_{i+1}^{x}+S_{i}^{y} S_{i+1}^{y}+S_{i}^{z} S_{i+1}^{z}\right)
$$

The $S=1 / 2$ Heisenberg antiferromagnetic (HAF) spin chain with the nearest-neighbor $(n n)$ interactions has a unique ground state with power-law correlations and the gapless excitation spectrum [39]. Using a concept of the valence bonds, this ground state has a character of a 
resonating-valence-bond (RVB) state, in which the quantum fluctuations restore the translational symmetry and mix in the bonds of greater length than those connecting only the $n n$ spins [40]. An alternative approach is the mapping of the quantum spin $1 / 2$ HAF chain to a chain of interacting spinless fermions [26]. The absence of the fermion (hole) at a site $i$ means a spin state $\left|S_{i}^{z}=-\frac{1}{2}\right\rangle$, whereas the presence of the fermion (particle) means $\left|S_{i}^{z}=\frac{1}{2}\right\rangle$. In this language, the model becomes a realization of the 1D Tomonaga-Luttinger liquid [41]. A corresponding ground state has correlations decreasing as a power law with a distance and elementary excitations form a particle-hole continuum. The excitation of a single hole carrying spin $1 / 2$ is called a spinon and can be created by a turning half of the chain upside down which results in the two neighboring sites with the spin up. The $x-y$ part of the Hamiltonian in the Equation (3) moves the spinon by two lattice sites (Figure 1).

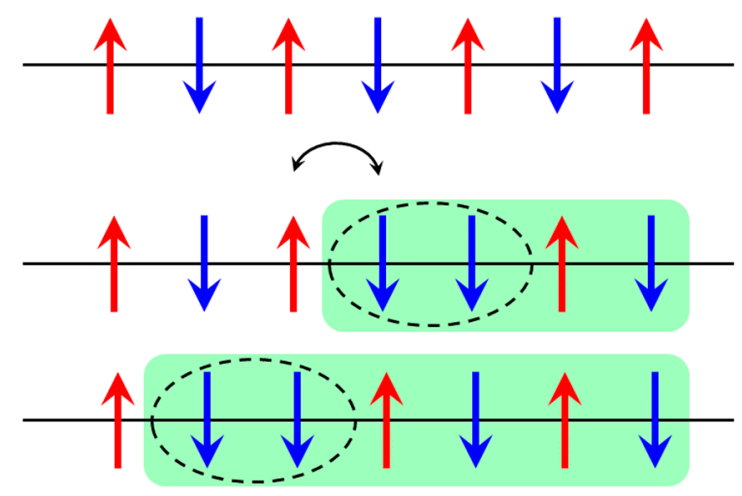

Figure 1. Schematic representation of a spinon excitation by reversing all spins beyond a certain lattice site. The spinon (domain wall) propagates along the chain moving by two lattice sites.

On the other hand, a standard magnon excitation carrying spin 1, is created by a flipping single spin at a lattice site. It corresponds to the two holes in the spectrum. As a consequence of the Fermion nature of the half-integer spins, the spin 1 carried by the spin wave excitation is decomposed into the pair of the $S=1 / 2$ quantum solitons, known as spinons [41]. The pairs of spinons form a gapless continuum of excitations experimentally observed in various quasi-low dimensional magnets. One example of such material is $\mathrm{KCuF}_{3}$ with strong $\mathrm{AF}$ exchange coupling propagating along the $c$-direction, while much weaker ferromagnetic coupling along the $a$ and $b$ directions is responsible for the onset of the long-range order at $T_{N}=39 \mathrm{~K}$. The inelastic neutron scattering measurements in zero magnetic field probed the energy spectrum of $\mathrm{KCuF}_{3}$ below and above $T_{\mathrm{N}}$. In comparison with low temperatures, the measurements made at $50 \mathrm{~K}$ indicated only little change in the scattering cross-section. The calculated spectrum of the $S=1 / 2 \mathrm{HAF}$ chain is in good agreement with the experimental data [42] (Figure 2).

While in $\mathrm{KCuF}_{3}$ the inter-chain coupling, $J^{\prime}$, is rather weak $\left(J^{\prime} \approx 0.01 J\right)$, in $\mathrm{Cs}_{2} \mathrm{CuCl}_{4}$, the $J^{\prime}$ achieves nearly $20 \%$ of the intra-chain value, $J / k_{\mathrm{B}} \approx 4 \mathrm{~K}$, and the onset of $\mathrm{LRO}$ occurs at $T_{\mathrm{N}}=0.62 \mathrm{~K}$. The low $J$-value guarantees rather easy achievement of the critical magnetic field, $B_{\mathrm{C}}$, necessary for decoupling the spin chains as well as the saturation field, $B_{\text {sat }}$, above which the ground state achieves a full ferromagnetic polarization. Theoretical studies of the $S=1 / 2$ HAF chain in the magnetic field [14] showed that the field splits the triplet excitation continuum into the separate continua, the positions of which alter with increasing field. Above the saturation field, the excitations have a character of well-defined magnon dispersion. Correspondingly, in $\mathrm{Cs}_{2} \mathrm{CuCl}_{4}$, the $1 \mathrm{D}$ regime was expected to set at the fields lower than $B_{\mathrm{sat}} \approx 6 \mathrm{~T}$. The magnetic excitations were studied as a function of the field by measuring the inelastic scattering at low temperatures, $T=0.06 \mathrm{~K}$ [43]. It was found that the intensity of the magnetic excitation decreases with increasing field and the line shape changes above $B_{\mathrm{c}}=1.66 \mathrm{~T}$, where the $1 \mathrm{D}$ regime occurs. Corresponding spectra were found to be in good agreement with the predictions for the $S=1 / 2 \mathrm{HAF}$ chain in the magnetic field [43]. 


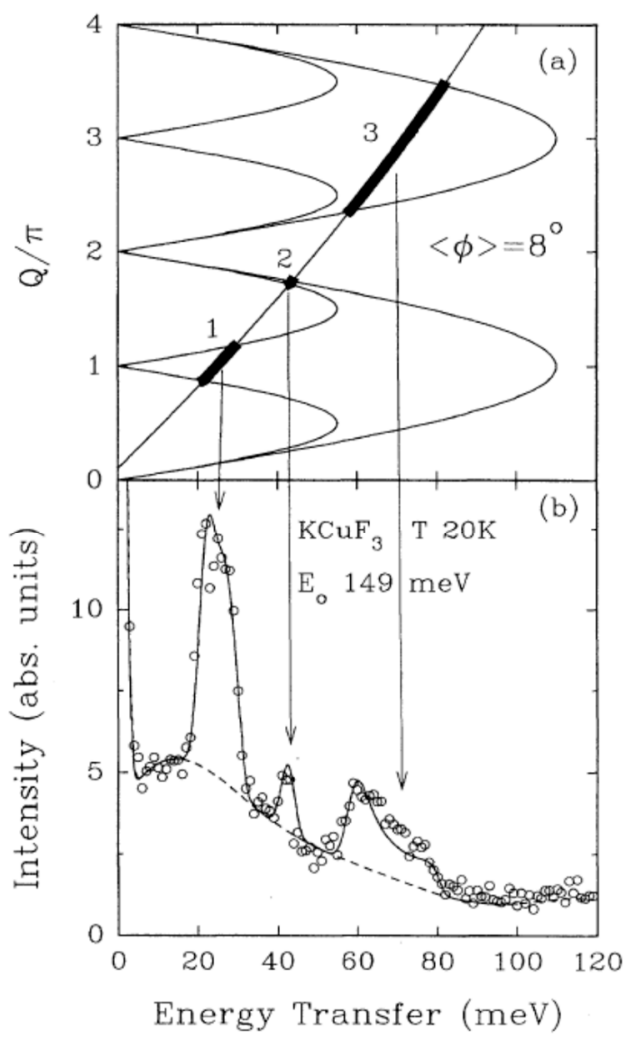

Figure 2. (a) Spectrum of the $S=1 / 2 \mathrm{HAF}$ chain in zero magnetic field. The thin solid line represents a scattering trajectory for a detector at the scattering angle of $8^{\prime}$ and an incident energy of $E_{o}=149 \mathrm{meV}$ in $\mathrm{KCuF}_{3}$. The scattering occurs when the trajectory intersects with the continuum (bold line); (b) Scattering measured in the low-angle detector banks at $T=20 \mathrm{~K}$. (Reproduced with permission from reference [42]).

The existence of the two-spinon continuum was experimentally confirmed in the organometallic compound $\mathrm{Cu}\left(\mathrm{C}_{4} \mathrm{H}_{4} \mathrm{~N}_{2}\right)\left(\mathrm{NO}_{3}\right)_{2}$, which proved to be an excellent realization of the $S=1 / 2 \mathrm{HAF}$ chain with $J=0.9 \mathrm{meV}$ and a negligible inter-chain coupling $\left(J^{\prime} / J<10^{-4}\right)$ [44].

These rather demanding neutron scattering studies are usually preceded by more accessible experimental techniques providing information on the finite-temperature macroscopic properties as specific heat, susceptibility and magnetization which serve as an important tool for the reliable identification of the magnetic system. For that purpose, many theoretical studies of the $S=1 / 2$ HAF chain based on different methods were performed to yield theoretical predictions usable for the analysis of experimental results [45-48]. The studies of real compounds approximating the model of the $S=1 / 2$ HAF chain point at the importance of additional exchange couplings, responsible for the deviations from the ideal chain behavior [13,42-44,49].

\subsection{The $S=1 / 2$ Heisenberg Antiferromagnet on the Spatially Anisotropic Square Lattice}

The absence of the Neel order in the ground state is believed to be a general feature of one-dimensional isotropic antiferromagnets $[17,18,41]$. Inter-chain coupling can change the ground-state properties and introduce dimensional crossover phenomena. A two-dimensional array of the spin chains coupled by the inter-chain interaction as depicted in Figure 3, forms a spatially anisotropic square lattice, often called as a rectangular lattice, which can be described by the Hamiltonian

$$
H=J\left[\sum_{\langle i, j\rangle_{J}} S_{i, j}, S_{i+1, j}+R \sum_{\langle i, j\rangle_{J^{\prime}}} S_{i, j} S_{i, j+1}\right] .
$$


The parameter $R=J^{\prime} / J$ represents the ratio of the AF inter-chain to AF intra-chain coupling and $\langle i, j\rangle_{J, J^{\prime}}$ denotes the nearest neighbors along the chain and perpendicular to the chain direction (Figure 3). For $R=0$, the 2D model (Equation (4)) simply reduces to the isolated $S=1 / 2 \mathrm{HAF}$ chains (Equation (3)) with the zero value of the order parameter (staggered magnetization), $m=0$, reflecting the absence of the LRO in the ground state. For $R=1$, Equation (4) represents the model of HAF on the spatially isotropic square lattice (or simply square lattice). According to Mermin-Wagner theorem [4] thermal fluctuations are strong enough to destroy the Néel LRO at finite temperatures. However, it was not clear, whether also quantum fluctuations can destroy the Néel LRO at zero temperature.

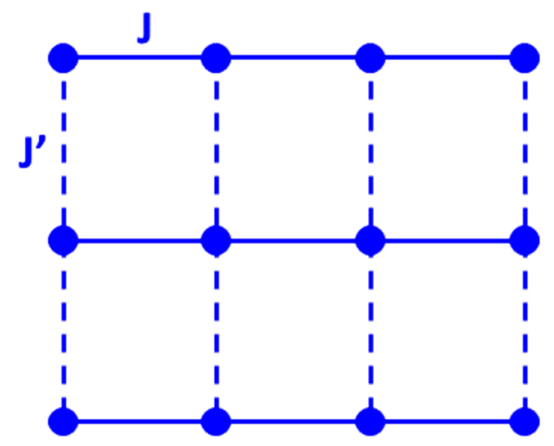

Figure 3. Cartoon of the spatially anisotropic square (三 rectangular) lattice.

After intensive studies of the ground-state properties of the spin $1 / 2$ HAF square lattice, over many decades a final agreement was achieved about the semi-classical Néel LRO at zero temperature [26]. The quantum fluctuations lead to the significant reduction of the order parameter, $m \approx 0.3$, which amounts about $60 \%$ of the classical value, $m_{0}=1 / 2$. The quantum fluctuations are strong enough to preserve spin-rotation symmetry such as the RVB state which may be relevant at high energies. While low-energy excitations are gapless magnons, recent experimental and theoretical studies showed that at higher energies, the existence of pairs of fractional $S=1 / 2$ quasiparticles, $2 \mathrm{D}$ analogs of $1 \mathrm{D}$ spinons was established [50]. Using various theoretical approaches, excitation spectra and finite-temperature properties of the square lattice were investigated including specific heat, uniform and staggered susceptibility, correlation length etc. to provide useful tools for the identification of the model realization in the real world [51-55]. In these studies, the compound $\mathrm{La}_{2} \mathrm{CuO}_{4}$, with the exchange coupling $J / k_{\mathrm{B}} \approx 1500 \mathrm{~K}$ proved to be a model system for the testing of the theories, especially those investigating ground-state properties. Naturally, the huge intra-layer coupling prevented any studies of the compound at moderate temperatures, $T \approx J / k_{\mathrm{B}}$. Later, other $2 \mathrm{D}$ magnetic systems, mostly copper(II) coordination complexes, were identified as excellent realizations of the spin $1 / 2 \mathrm{HAF}$ square lattice with much lower exchange coupling. The analysis of the specific heat of $\mathrm{Cu}(\mathrm{en})_{2} \mathrm{Ni}(\mathrm{CN})_{4}$ (en $\left.=\mathrm{C}_{2} \mathrm{H}_{8} \mathrm{~N}_{2}\right)$ and $\mathrm{Cu}(\mathrm{bmen})_{2} \mathrm{Pd}(\mathrm{CN})_{4}\left(\mathrm{bmen}=\mathrm{N}, \mathrm{N}^{\prime}\right.$-dimethyl-1,2-diaminoethane), revealed excellent agreement with the theoretical prediction for the spin $1 / 2 \mathrm{HAF}$ square lattice with $J / k_{\mathrm{B}}=0.36 \mathrm{~K}$ and $0.48 \mathrm{~K}$, respectively [56,57]. Unlike $\mathrm{La}_{2} \mathrm{CuO}_{4}$, small coupling and corresponding saturation field $B_{\text {sat }} \approx 1 \mathrm{~T}$, enable comfortable studies in the wide region of temperatures and magnetic fields. In these octahedral $\mathrm{Cu}$ (II) complexes comprising of weakly bound electroneutral covalent chains, the exchange coupling between $\mathrm{Cu}(\mathrm{II})$ ions is mediated predominantly through hydrogen bonds. On the other hand, in the tetragonal compound $\left[\mathrm{Cu}(\mathrm{pz})_{2}\left(\mathrm{NO}_{3}\right)\right]\left[\mathrm{PF}_{6}\right](\mathrm{pz}=$ pyrazine $)$ and monoclinic $\mathrm{Cu}(\mathrm{pz})_{2}\left(\mathrm{ClO}_{4}\right)_{2}$, the copper sites are connected within the square layers by bridging pyrazine molecules with the exchange coupling $J / k_{\mathrm{B}}=10.5 \mathrm{~K}$ and $17.5 \mathrm{~K}$, respectively [58,59]. Even larger exchange coupling was indicated in $\mathrm{Cu}(\mathrm{HCOO})_{2} \cdot 4 \mathrm{H}_{2} \mathrm{O}$ with $J / k_{\mathrm{B}}=72 \mathrm{~K}$ [60]. Magneto-structural investigations [61-63] of monoclinic compounds (5MAP) ${ }_{2} \mathrm{CuBr}_{4}$ and $(5 \mathrm{BAP})_{2} \mathrm{CuBr}_{4}$ (5MAP $=5$-methyl-2-aminopyridinium, $5 \mathrm{BAP}=5$-bromo-2-aminopyridinium) revealed that the magnetic interaction occurs between $\mathrm{Cu}(\mathrm{II})$ sites with four equivalent nearest neighbors through $\mathrm{Br} \cdots \mathrm{Br}$ contacts forming 2D square layers with exchange coupling $J / k_{\mathrm{B}} \approx 7 \mathrm{~K}$. The layers of $\mathrm{CuBr}_{4}$ tetrahedrons are separated by the bulk of organic 
cations which stabilize 3D structure. Systematic study of the compounds from the series $\mathrm{A}_{2} \mathrm{CuX}$ [A $=5 \mathrm{MAP}, 5 \mathrm{BAP}$, 5-chloro-2-aminopyridinium $\equiv 5 \mathrm{CAP}$, 5-cyano-2-aminopyridinium $\equiv 5 \mathrm{CNAP}$, etc., $\mathrm{X}=\mathrm{Br}, \mathrm{Cl}$ ] found that the increasing size of the A cation improves the isolation of individual magnetic square layers but at the same time it reduces the strength of the intra-layer exchange coupling [62-64]. Apparently, the chemical modification of the structure can control magnetic properties demonstrating the flexibility of molecular magnetism.

Intensive theoretical studies of the ground-state and finite-temperature properties of the square lattice $(R=1)$ provided reach variety of theoretical predictions allowing reliable identification of real candidates. Besides the materials manifesting the model realization of the $S=1 / 2$ HAF square lattice, there appeared two-dimensional quantum systems with some amount of the spatial anisotropy of the intra-layer exchange coupling which could not be well determined due to the lack of proper theoretical predictions [65-70]. Using various approaches, the ground-state properties of the spatially anisotropic square lattice (Equation (4)) were investigated in the vicinity of the isotropic model $(R=1)$ and $1 \mathrm{D}$ chain $(R=0)$. Many theoretical studies tried to solve the question about the existence of a critical $R_{\mathrm{c}}$ value above which a long-range order is established in the ground state $(m>0)$. Conventional spin-wave theories as well as various numerical techniques predicted a final value, $R_{\mathrm{c}} \approx 0.1-0.2$, below which a $2 \mathrm{D}$ spin-liquid state with $m=0$ can be stabilized [71,72]. For small $R$, a single-chain mean-field theory [73] predicted $R_{\mathrm{c}}=0$ and a gradual increase of $m$, proportional to $\sqrt{R}$. Multi-chain mean-field calculations complemented by large-scale Monte Carlo simulations of the 2D Hamiltonian (Equation (4)), confirmed that $R_{\mathrm{c}}=0$ and showed that for $R \rightarrow 0, m$ vanishes slower than $\sqrt{R}$ due to a logarithmic correction to this form [74]. Applying various techniques [72], the order-disorder ground-state transition was indicated for $R \approx 0.2$ (Figure 4a). The sharp change of $m$ for $R<0.2$ was interpreted as a crossover in the magnetic behavior of the $S=1 / 2$ HAF rectangular lattice, accompanied by a sharp change in the spatial dependence of spin correlations; with decreasing $R$, the rising quantum fluctuations gradually reduce the size of the order parameter and for small $R$, the system approaches $1 \mathrm{D}$ behavior with algebraic decrease of correlation functions [72]. This conclusion was further supported by quantum Monte Carlo studies [75] of finite-temperature properties of the rectangular lattice (Equation (4)). For small $R$, the temperature dependence of the uniform susceptibility, $\chi$, follows that of a single chain, while deviations appear below temperatures $k_{\mathrm{B}} T / J \approx 5 R$. For larger $R$, the $\chi$ values lie between those of the chain and square lattice. The 1D - 2D dimensional crossover is evident also in the behavior of the correlation length, $\xi$, depicted in Figure $4 \mathrm{~b}$. For $R>0$ and low temperatures, the quantity is well described by the relation [75]

$$
\xi=\frac{A \exp \left[2 \pi \rho_{s}(R) /\left(k_{B} T\right)\right]}{1+0.5 T /\left[2 \pi \rho_{s}(R)\right]}
$$

where the spin stiffness, $\rho_{s}$, depends on the spatial anisotropy $R$. As can be seen in Figure $4 \mathrm{~b}$, the intra-chain correlation length gradually approaches $1 \mathrm{D}$ behavior when decreasing $R$. Alike susceptibility, for small $R$, the $\xi$ values merge with those for a single chain at sufficiently high temperatures. Using these theoretical predictions, authors of reference [75] identified the quantum magnet $\mathrm{Sr}_{2} \mathrm{CuO}_{3}$ as a realization of the $S=1 / 2 \mathrm{HAF}$ on the rectangular lattice with the intra-chain coupling $J / k_{\mathrm{B}}=2200 \mathrm{~K}, R=0.002$ and the staggered magnetization, $m \approx 0.03$. In comparison with the $m \approx 0.3$ derived for the square lattice, the significant reduction results from the strong enhancement of quantum fluctuations.

While the ground-state properties of the rectangular lattice were already understood quite well, corresponding finite-temperature studies appeared only recently [76,77]. Quantum Monte Carlo simulations of the susceptibility and magnetization enabled to identify the realizations of the $S=1 / 2$ $\mathrm{HAF}$ rectangular lattice, namely $\mathrm{Cu}(\mathrm{pz}) \mathrm{Cl}_{2}\left(J / k_{\mathrm{B}}=28 \mathrm{~K}, R=0.3\right), \mathrm{Cu}(\mathrm{pz})\left(\mathrm{N}_{3}\right)_{2}\left(J / k_{\mathrm{B}}=15 \mathrm{~K}, R=0.46\right)$ and $\mathrm{Cu}\left(2\right.$-apm) $\mathrm{Cl}_{2}$ (2-apm $=2$-amino-pyrimidine) with $\mathrm{J} / k_{\mathrm{B}}=116.3 \mathrm{~K}$ and $R=0.084$ [77].

It should be noted that a reliable identification of a real compound requires rather complex and careful analysis of bulk properties. It proved that making final conclusion on the basis of even several experimental techniques cannot be sufficient since various models can provide the same theoretical 
prediction. In that case, first-principle calculations can provide valuable information how to proceed in further analysis as was demonstrated in the analysis of the susceptibility of $\mathrm{Cu}(\mathrm{NCS})_{2}$ where the first-principle calculations enabled to identify the material as the realization of the rectangular lattice with $J / k_{\mathrm{B}} \approx 170 \mathrm{~K}$ and $R \approx 0.08$ [78].

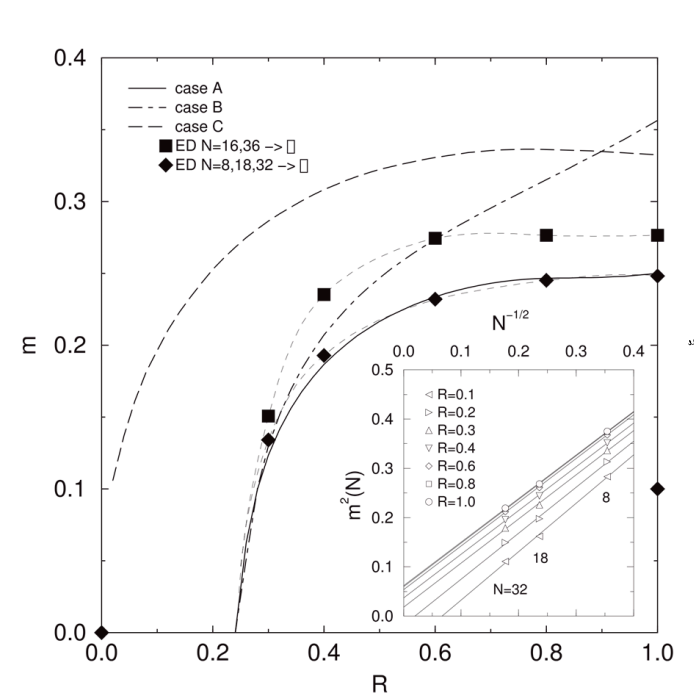

(a)

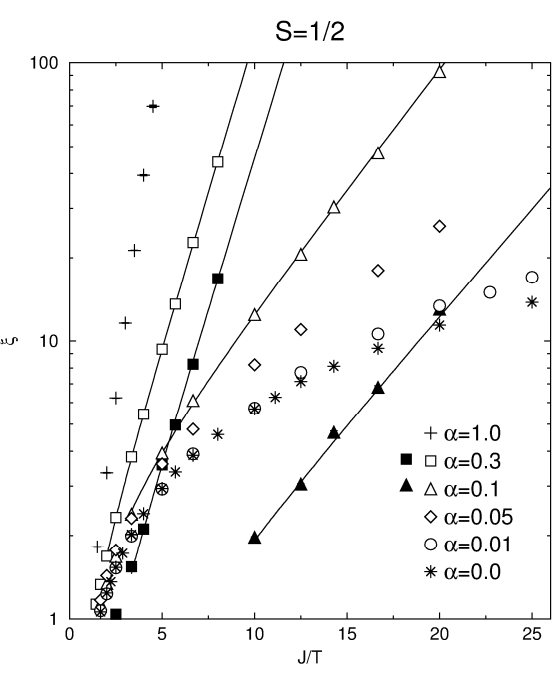

(b)

Figure 4. (a) Ground-state staggered magnetization $m$ of the rectangular lattice for different $R$ values calculated by various approaches; the short-dashed lines are fits to the exact diagonalization (ED) data. Cases $A, B, C$ correspond to different choice of parameters in Green's function approach. The inset demonstrates the deviation of the ED results from the N-1/2 scaling law at $R<0.3$ (solid lines are least-squares fits). (Reproduced with permission from reference [72]); (b) The correlation length for the $S=1 / 2 \mathrm{HAF}$ on the rectangular lattice as a function of the inverse temperature for various $R$ values ( $\alpha$ stands for $R$ ). Open (filled) symbols denote the correlation length along (perpendicular to) the chain. For small $\alpha(\alpha<0.05)$, the values of inter-chain correlation length are smaller than one lattice constant and are not shown in the figure. Solid lines are fits to Equation (5), showing the exponential dependence of $\xi$ on $1 / T$. (Reproduced with permission from reference [75]).

Similarly, it was shown in reference [79], that the susceptibility and isothermal magnetization of $\mathrm{Cu}(\mathrm{PM})(\mathrm{EA})_{2}\left(\mathrm{PM}=\left[\mathrm{C}_{6} \mathrm{H}_{2}(\mathrm{COO})_{4}\right]^{4-}, \mathrm{EA}=\left[\mathrm{C}_{2} \mathrm{H}_{5} \mathrm{NH}_{3}\right]^{+}\right)$can be described by the model (Equation (4)) with various $R$ in a wide range of temperatures and magnetic fields (Figure 5).

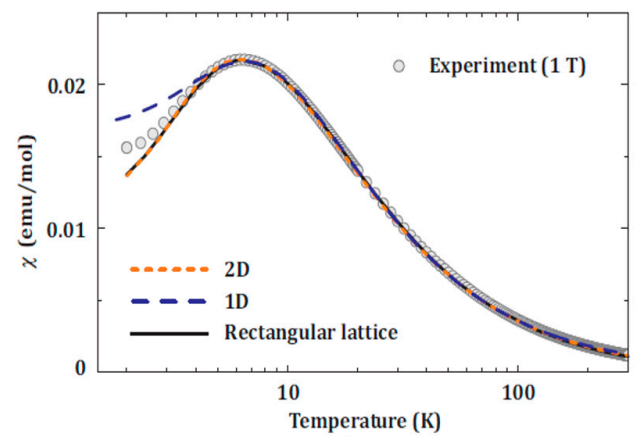

(a)

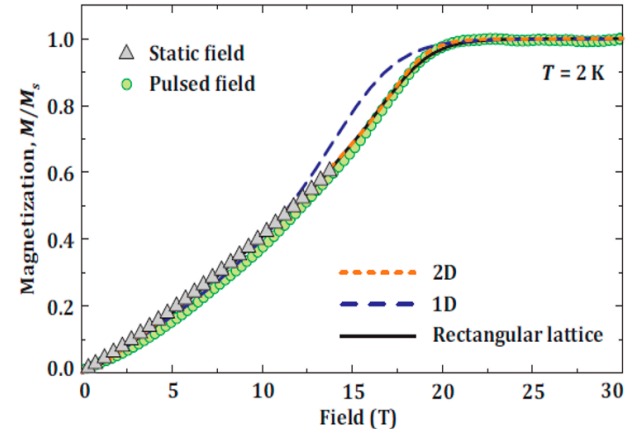

(b)

Figure 5. (a) Fits of the magnetic susceptibility of $\mathrm{Cu}(\mathrm{PM})(\mathrm{EA})_{2}$ with the model (Equation (4)) for $R=0$ (1D) with $J / k_{\mathrm{B}}=10.2 \mathrm{~K}, g=2 ; R=1$ (2D) with $J / k_{\mathrm{B}}=6.8 \mathrm{~K}, g=2.05 ; R=0.7$ (rectangular lattice) with $J / k_{\mathrm{B}}=8.0 \mathrm{~K}, g=2.07$; $(\mathbf{b})$ Field dependence of the magnetization of $\mathrm{Cu}(\mathrm{PM})(\mathrm{EA})_{2}$. Lines show the simulations with the same parameters as those used for the susceptibility. (Reproduced with permission from reference [79]). 
Apparently, using proper parameters, various theoretical predictions yield identical behavior for both, susceptibility and magnetization [79]. Thus, making conclusions on the basis of magnetic data only, can be very tricky, since the height of the maximum, $\chi_{\max }$, as well as its position, $T_{\max }$, depend on the $J$ and $g$-factor values. Correspondingly, the proper choice of $g$ and $J$ parameters can yield an excellent agreement with the data (Figure 5). Unlike the susceptibility, another bulk property, specific heat, can provide more valuable information since the maximum, $C_{\max }$, depends on the used model (Figure 6). The best agreement was found for $R=0.7$ as predicted by the first-principle calculations [79].

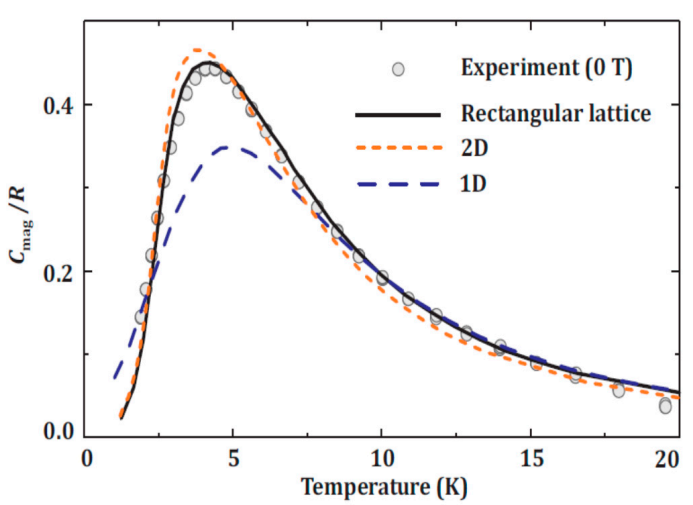

(a)

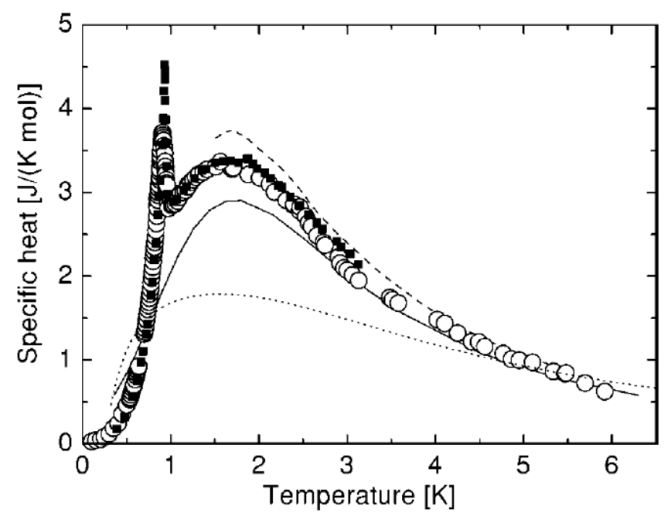

(b)

Figure 6. (a) Fits of the magnetic specific heat of $\mathrm{Cu}(\mathrm{PM})(\mathrm{EA})_{2}$ in $B=0$ with the model (Equation (4)) for $R=0$ (1D), $R=1$ (2D) and $R=0.7$ (rectangular lattice) with the same parameters as in Figure 5. (Reproduced with permission from reference [79]); (b) Fits of the magnetic specific heat of $\mathrm{Cu}(\mathrm{en})\left(\mathrm{H}_{2} \mathrm{O}\right)_{2} \mathrm{SO}_{4}$ in $B=0$ (open and full circles correspond to powder and single crystal, respectively) with the model (Equation (4)) for $R=0$ with $J / k_{\mathrm{B}}=3.6 \mathrm{~K}$, (solid line), $R=1$ with $J / k_{\mathrm{B}}=2.8 \mathrm{~K}$ (dashed line) and the model of the $S=1 / 2 \mathrm{HAF}$ on the triangular lattice with $J / k_{\mathrm{B}}=4.4 \mathrm{~K}$ (dotted line). (Reproduced with permission from reference [65]).

Such calculations proved to be very important in the identification of the 2D quantum magnet $\mathrm{Cu}(\mathrm{en})\left(\mathrm{H}_{2} \mathrm{O}\right)_{2} \mathrm{SO}_{4}$. Previous analysis of powder thermodynamic data [65] identified the material as a potential realization of the partially frustrated $S=1 / 2 \mathrm{HAF}$ on the spatially anisotropic triangular lattice (SATL). The lack of proper theoretical predictions enabled the analysis only in the frame of the limiting models of the SATL, i.e., the square lattice, chain and triangular lattice and could not provide a reliable information whether the spin system approaches the properties of the chain or the square lattice (Figure $6 \mathrm{~b}$ ). Considering $d_{\mathrm{x}}{ }^{2}-\mathrm{y}^{2}$ ground state of the $\mathrm{Cu}$ (II) ion, it was assumed that potential exchange pathways form SATL with a dominant exchange coupling creating the square lattice, while weaker interactions were expected to occur along one of the diagonals of the square plaquettes [65]. The analysis of single-crystal electron paramagnetic resonance spectra [80] indicated the need to revisit the concept of SATL in $\mathrm{Cu}(\mathrm{en})\left(\mathrm{H}_{2} \mathrm{O}\right)_{2} \mathrm{SO}_{4}$, which triggered first-principle calculations of exchange couplings [81]. The calculations revealed the formation of a spatially anisotropic zig-zag square lattice (Figure 7a) comprised of 2D array of weakly coupled zig-zag chains with $R=J^{\prime} / J \approx 0.15$. Corresponding quantum Monte Carlo (QMC) calculations of finite-temperature properties of the $S=1 / 2$ HAF on the spatially anisotropic zig-zag square lattice (SAZZSL) including specific heat, susceptibility and magnetization, provided theoretical predictions in a wide range of temperatures and magnetic fields [82]. Subsequent analysis of single-crystal $\mathrm{Cu}(\mathrm{en})\left(\mathrm{H}_{2} \mathrm{O}\right)_{2} \mathrm{SO}_{4}$ experimental data within the SAZZSL model found the excellent agreement for $J / k_{\mathrm{B}}=3.4 \mathrm{~K}$ and $R=0.35$ (Figure 8).

The comparison of the finite-temperature properties of the $S=1 / 2 \mathrm{HAF}$ on the rectangular and zig-zag square lattice surprisingly revealed the identical behavior in the whole range of the spatial anisotropy $R[77,82]$. Thus, to decide which model is appropriate for the description of the real compound, first-principle studies are very important. 


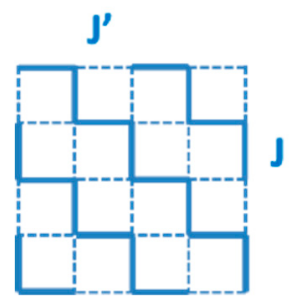

(a)

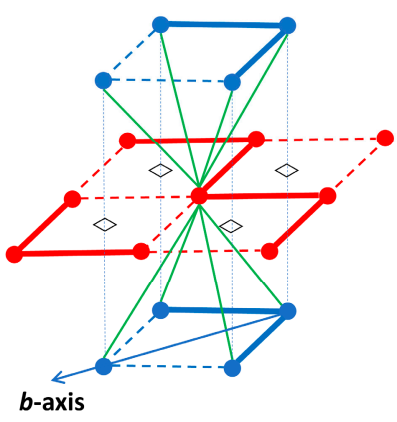

(b)

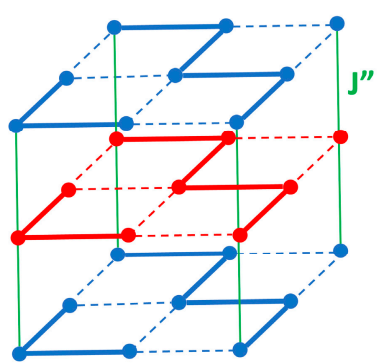

(c)

Figure 7. (a) Spatially anisotropic zig-zag square lattice interpolates between the chain $\left(R=J^{\prime} / J=0\right)$ and the isotropic square lattice $(R=1)$; (b) Position of adjacent layers (blue) in $\mathrm{Cu}(\mathrm{en})\left(\mathrm{H}_{2} \mathrm{O}\right)_{2} \mathrm{SO}_{4}$ with respect to the central 2D layer (red). Diamonds denote projections of the positions of blue circles into the central layer; (c) Position of adjacent layers (blue) in the verdazyl radical with respect to the central 2D layer (red). In (b) and (c), the inter-layer exchange couplings $J^{\prime \prime}$ are depicted by green lines (see Section 2.3).

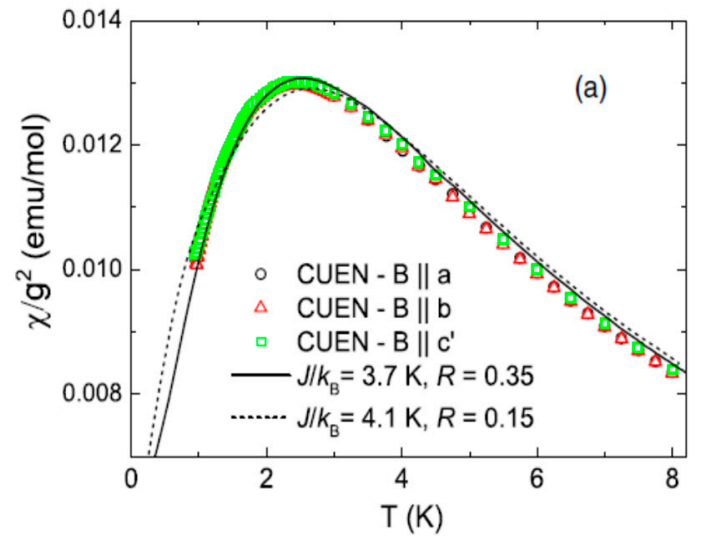

(a)

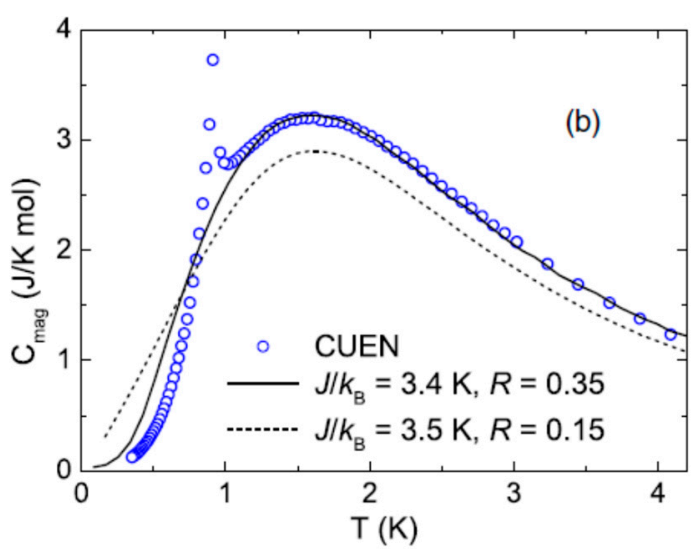

(b)

Figure 8. (a) Temperature dependence of normalized single-crystal $\mathrm{Cu}(\mathrm{en})\left(\mathrm{H}_{2} \mathrm{O}\right)_{2} \mathrm{SO}_{4}(\mathrm{CUEN})$ susceptibility in the field $10 \mathrm{mT}$. Solid and dashed lines represent corresponding QMC calculations ( $L=128$ ) for the $S=1 / 2$ HAF SAZZSL model with $R=0.35$ and 0.15 , respectively; (b) Temperature dependence of magnetic specific heat of CUEN single crystal in zero magnetic field. Solid and dashed lines have the same meaning as in (a). (Reproduced with permission from reference [82]).

As was already mentioned, the compound $\mathrm{Cu}(\mathrm{pz})_{2}\left(\mathrm{ClO}_{4}\right)_{2}$ was considered as a model system for the realization of the isotropic square lattice [59]. Recent density functional theory (DFT) simulations using structural $\mathrm{Cu}(\mathrm{pz})_{2}\left(\mathrm{ClO}_{4}\right)_{2}$ data at $10 \mathrm{~K}$ revealed the formation of SAZZSL with $R=0.7$ [83] while other authors working with $100 \mathrm{~K}$ structural data arrived to the rectangular lattice with $R \approx 0.93$ [84]. While it is hard to discriminate between two different results on the basis of magnetic bulk data, as was done in refs. [83,84], specific heat analysis would be subservient to make the decision. As was shown in refs. [79,82], the difference in the maximum specific heat values for $R=1$ and 0.7 is easily distinguishable. This approach was applied also in the determination of the 2D magnetic lattice in the verdazyl radical $\alpha-2,3,5-\mathrm{Cl}_{3}-\mathrm{V}$ (3-(2,3,5-trichlorophenyl)-1,5-diphenylverdazyl] (Figure 7) where DFT calculations revealed the formation of SAZZSL with $R=0.56$ [85].

All these results indicate, that rather simple combination of bulk properties and first-principle calculations can provide a reliable identification of magnetic lattice in the real compounds. However, unlike the ideal 2D models, in real compounds, the interlayer interactions are always present and depending on their strength, they can affect bulk properties, usually leading to the onset of the 3D long-range order. 


\subsection{The Crossover from $2 D$ to $3 D$}

The need to understand the effect of the inter-layer coupling, $J^{\prime \prime}$, on the properties of two-dimensional magnets stimulated theoretical studies of the 3D array of coupled layers with the square-lattice motif described by the Equation (4), forming the $S=1 / 2 \mathrm{HAF}$ on the spatially anisotropic simple cubic lattice [51,86-88]. The studies showed that the coupling between the magnetic layers can induce a phase transition to 3D LRO at a finite temperature [51]

$$
k_{B} T_{N} \approx J^{\prime \prime}\left(\frac{m}{m_{0}}\right)^{2}\left(\frac{\xi}{a}\right)^{2}
$$

where $a$ is a lattice constant, $m / m_{0}$ is the reduced staggered magnetization at zero temperature, and correlation length is defined by the Equation (5). For the isotropic square lattice $(R=1),\left(\mathrm{m} / \mathrm{m}_{0}\right)^{2} \approx$ 0.3 and the spin stiffness in the Equation (5) is $\rho_{\mathrm{s}} \approx 0.18 \mathrm{~J}$. Apparently, even a minute amount of $J^{\prime \prime}$ is capable to induce a 3D LRO at a finite temperature. Large-scale QMC studies of the $S=1 / 2 \mathrm{HAF}$ on the spatially anisotropic simple cubic lattice (with $R=1$ within layers) provided an empirical formula for $T_{\mathrm{N}}$ which enables the estimation of the inter-layer coupling [88]

$$
T_{N}=4 \pi \rho_{s} /\left[b-\ln \left(J^{\prime \prime} / J\right)\right]
$$

with $\rho_{\mathrm{s}}=0.183 \mathrm{~J}$ and $b=2.43$.

As was already mentioned, in the absence of $J$ " the ground-state staggered magnetization vanishes with $R \rightarrow 0$ (Figure 4a). On the other hand, the increase of $J$ " leads to the enhancement of $m$ in the whole range of $R$ [86]. Thus, the inclusion of the un-frustrated interlayer coupling stabilizes a collinear Néel order already for very small values of $J^{\prime \prime}$. The competition of the inter-layer coupling and the intra-layer spatial anisotropy $R$ projects also to the finite-temperature properties [86]; for $R \rightarrow 0$ within the layer, enhanced quantum fluctuations lead to reducing $T_{N}$. On the other hand, nonzero $J^{\prime \prime}$ reduces the strength of the quantum fluctuations, which results in the enhancement of $T_{\mathrm{N}}$. In highly anisotropic 2D systems $\left(J^{\prime \prime} " J, J^{\prime}\right)$, most of the entropy is removed above $T_{\mathrm{N}}$ and the effective number of degrees of freedom associated with the 3D LRO is significantly reduced. In such extreme conditions, a $\lambda$-like anomaly in the specific heat associated with the onset of 3D LRO completely vanishes. Quantum Monte Carlo studies of the $S=1 / 2$ HAF on the spatially anisotropic simple cubic lattice ( $R=1$ within layers) showed, that a sharp peak dominates the specific heat behavior for the strong inter-layer coupling, while for $J^{\prime \prime}<0.05 J$ a clear separation of two peaks occurs [87]. Finally, for $J^{\prime \prime}<0.015 J$, the sharp peak completely vanishes and despite the onset of $3 \mathrm{D}$ LRO, the specific heat follows the behavior of the $2 \mathrm{D}$ system (Figure 9a).

Such extreme spatial two-dimensionality accompanied by the absence of the $\lambda$-like anomaly in the specific heat in zero magnetic field was already experimentally observed in $\mathrm{Cu}(\mathrm{tn}) \mathrm{Cl}_{2}$ (tn $=\mathrm{C}_{3} \mathrm{H}_{10} \mathrm{~N}_{2}$ ) [89] (Figure 9b) and $\mathrm{Cu}(\mathrm{pz})_{2}(\mathrm{pyO})_{2}\left(\mathrm{PF}_{6}\right)_{2}(\mathrm{pyO}=$ pyridine-N-oxide) [90]. Thus, one has to be careful in the declaration of the absence of the 3D LRO on the basis of the specific heat only and other experiments are necessary to confirm the assumption. Besides demanding neutron diffraction experiments, much simpler susceptibility measurements or electron paramagnetic-antiferromagnetic resonance can provide reliable information about the onset of the 3D LRO. Neglecting any other phenomena but $J^{\prime \prime}$, the absence or the presence of the $\lambda$-like anomaly can be affected by the geometry of inter-layer exchange pathways in real compounds. The comparison of magnetic specific heats of $\mathrm{Cu}(\mathrm{tn}) \mathrm{Cl}_{2}$ and $\mathrm{Cu}(\mathrm{en})\left(\mathrm{H}_{2} \mathrm{O}\right)_{2} \mathrm{SO}_{4}$ suggests that the $2 \mathrm{D}$ correlations responsible for the appearance of a round maximum have the same character while the manifestation of the 3D correlations is completely different (Figure $9 \mathrm{~b}$ ). The severe weakening of their effect in $\mathrm{Cu}(\mathrm{tn}) \mathrm{Cl}_{2}$ was ascribed to the combined effect of a geometrical frustration and the large distances (about $10 \AA$ ) between $\mathrm{Cu}$ (II) ions in the adjacent layers [89].

On the other hand, the strength of the inter-layer coupling in $\mathrm{Cu}(\mathrm{en})\left(\mathrm{H}_{2} \mathrm{O}\right)_{2} \mathrm{SO}_{4}$ was estimated to be much lower than $0.015 \mathrm{~J}$ [82] thus, according to reference [87], no phase transition should be visible 
in the specific heat data. In $\mathrm{Cu}(\mathrm{en})\left(\mathrm{H}_{2} \mathrm{O}\right)_{2} \mathrm{SO}_{4}$, the adjacent layers are shifted along the $b$-axis by $b / 2$. As a consequence, a central spin from the magnetic layer has a high number of nearest neighbors $(z=8)$ from the adjacent layers (Figure 7). It should be noted, that QMC simulations [87] were performed for the geometry of the simple cubic lattice, thus only $z=2$ was considered for the central spins in the layer.

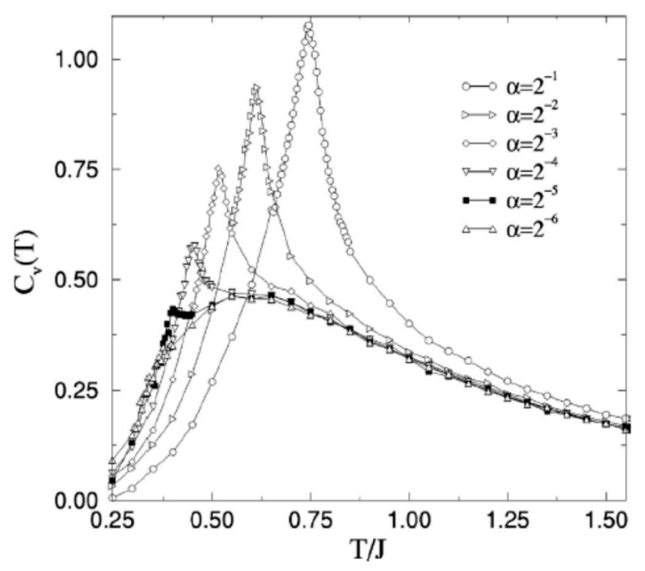

(a)

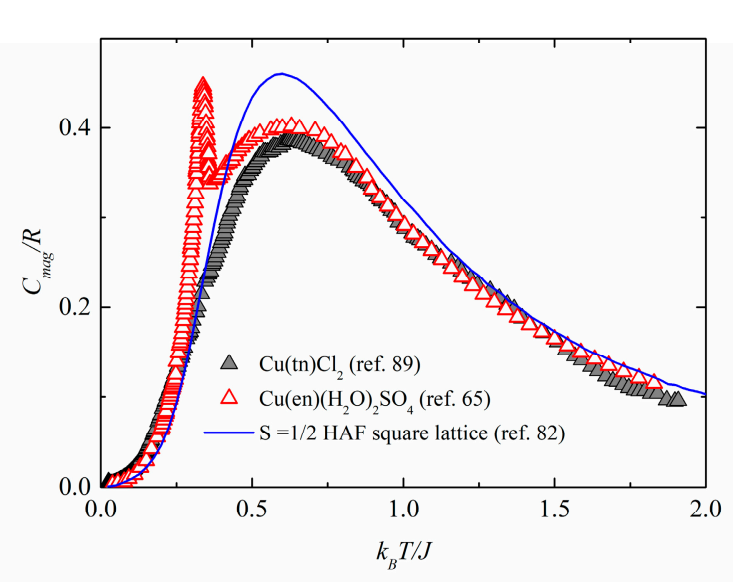

(b)

Figure 9. (a) The specific heat of the $S=1 / 2$ HAF model on the spatially anisotropic simple cubic lattice with $R=1$ within the layers. Quantum Monte Carlo calculations are performed over a wide temperature range for several different spatial anisotropies $\left(\alpha=J^{\prime \prime} / J\right)$. The system size is $48 \times 48 \times 12$. The separation of the 3D ordering peak from the broad maximum arising from the 2D physics is clearly visible for $\alpha<2^{-3}$. (Reproduced with permission from reference [87]); (b) Temperature dependence of $\mathrm{Cu}(\mathrm{en})\left(\mathrm{H}_{2} \mathrm{O}\right)_{2} \mathrm{SO}_{4}\left(J / k_{\mathrm{B}}=2.8 \mathrm{~K}\right)$ and $\mathrm{Cu}(\mathrm{tn}) \mathrm{Cl}_{2}\left(J / k_{\mathrm{B}}=3.1 \mathrm{~K}\right)$ magnetic specific heat compared with the theoretical prediction for the $S=1 / 2 \mathrm{HAF}$ on the square lattice $(R=1)$.

The important role of the number of neighbors in adjacent planes was demonstrated also in the verdazyl radical $\alpha-2,3,5-\mathrm{Cl}_{3}-\mathrm{V}$ with a large inter-layer coupling, $\mathrm{J}^{\prime \prime} \approx 0.24 \mathrm{~J}$. This is another extreme, since such a high value of $J^{\prime \prime}$ should completely suppress the two-dimensional character of the magnetic system in the verdazyl radical. However, thermodynamic properties clearly demonstrate a high measure of magnetic two-dimensionality, comparable to $\mathrm{Cu}(\mathrm{en})\left(\mathrm{H}_{2} \mathrm{O}\right)_{2} \mathrm{SO}_{4}$. This contradiction can be explained by very low number of interacting nearest neighbors from the adjacent planes. As can be seen in Figure 7, in verdazyl radical, effectively only $z=0.5$ per central spin can be considered, thus reducing the effect of rather strong inter-layer coupling can be expected [85].

Generally, if only isotropic inter-layer coupling is responsible for the onset of the 3D LRO, the finite-temperature phase transition in such spatially anisotropic systems remains in the universality class of the 3D classical Heisenberg model [87]. The universality can be changed when other mechanisms are present as the magnetic field and spin anisotropy [91].

\section{The Effect of the Spin Anisotropy and Magnetic Field in the $S=1 / 2 \mathrm{HAF}$ on the Square Lattice}

\section{1. $B=0$}

The influence of the spin-orbit (LS) coupling projects into the anisotropy of local as well as global magnetic properties. In the case of isolated ions with the electronic spin $1 / 2$ (e.g., $\mathrm{Cu}$ (II) ion with the $3 d^{9}$ open shell), the interplay of the LS coupling and the crystal field produced by the local surrounding generates the anisotropy of the magnetic moment, observed in the form of the anisotropic $g$-tensor [92]. In the presence of magnetic interactions between the paramagnetic ions, the exchange anisotropy (symmetric and/or antisymmetric) appears with the relative strength comparable with the order of the $g$-factor anisotropy [93]. Accordingly, experimental studies of quasi-2D spin 1/2 magnets revealed that 
the spin anisotropy is very weak, ranging from $10^{-4}$ to $10^{-2}$ times the intra-layer exchange coupling $J[59,80,94]$.

In case of the symmetric exchange anisotropy, theoretical studies of the $S=1 / 2$ antiferromagnetic XXZ model on the square lattice $(R=1)$ revealed, that even extremely weak spin anisotropies can influence thermodynamic properties at low temperatures, inducing a crossover between the isotropic Heisenberg and the anisotropic regime [95-97]. The $S=1 / 2$ AF XXZ model (i.e., anisotropic Heisenberg antiferromagnet) on the square lattice can be defined by the Hamiltonian [97]

$$
H=\frac{J}{2} \sum_{i, d}\left[\left(1-\Delta_{\mu}\right)\left(S_{i}^{x} S_{i+d}^{x}+S_{i}^{y} S_{i+d}^{y}\right)+\left(1-\Delta_{\lambda}\right) S_{i}^{z} S_{i+d}^{z}\right]
$$

where $i=\left(i_{1}, i_{2}\right)$ runs over the sites of the square lattice, $d$ connects the $i$-th site to the nearest neighbors, $J>0$ is antiferromagnetic coupling, $\Delta_{\mu}$ and $\Delta_{\lambda}$ are the easy-axis and easy-plane anisotropy parameters, respectively. For $\Delta_{\mu}=\Delta_{\lambda}=0$, the Equation (8) reduces to the Equation (4) with $R=1$ describing the isotropic HAF on the square lattice. The parameters $\Delta_{\lambda}=0,0<\Delta_{\mu} \leq 1$ define the easy-axis anisotropy, while the easy-plane anisotropy corresponds to $\Delta_{\mu}=0,0<\Delta_{\lambda} \leq 1$. In the case of the weak easy-axis anisotropy $\Delta_{\mu}=10^{-2}$ and $10^{-3}$, quantum Monte Carlo studies of finite-temperature properties revealed the existence of a phase transition in the $2 \mathrm{D}$ Ising universality class occurring at finite temperatures, $k_{\mathrm{B}} T_{\mathrm{I}} \approx 0.28 \mathrm{~J}$ and $0.22 \mathrm{~J}$, respectively [97]. In the specific heat, the onset of the 2D LRO was indicated as a small sharp peak superimposed on the left side of a round maximum. As the anisotropy decreased, the sharp peak diminished, moving to low temperatures, while the round maximum converged to that of the HAF on the square lattice. Similarly, the uniform susceptibility follows the prediction for the HAF on the square lattice down to $k_{\mathrm{B}} T \approx 0.4 \mathrm{~J}$ for $\Delta_{\mu}=10^{-2}$. At lower temperatures, the transverse susceptibility, $\chi^{\mathrm{xx}}$, and longitudinal, $\chi^{\mathrm{zz}}$, separate from the isotropic Heisenberg curve well above the phase transition; at the transition temperature $T_{\mathrm{I}}$, the $\chi^{\mathrm{xx}}$ displays a minimum, while $\chi^{\mathrm{zz}}$ monotonically decreases to zero. Apparently, the susceptibility measurement in two different orientations of magnetic field provides a tool for a reliable identification of the phase transition. Similar features of the susceptibility and specific heat were observed in the XXZ model with the easy-plane anisotropy for $\Delta_{\lambda}=2 \times 10^{-2}$ and $10^{-3}$. A crossover temperature, $T_{\mathrm{CO}}$, from the isotropic Heisenberg to the easy-plane (XY) behavior was estimated [96]

$$
\frac{k_{B} T_{C O}}{J} \cong \frac{4 \pi \rho_{s} / J}{\ln \left(C / \Delta_{\lambda}\right)}
$$

with the parameter $C=160$ and $\rho_{\mathrm{s}}=0.214 \mathrm{~J}$. The onset of the $\mathrm{XY}$ regime below $T_{\mathrm{CO}}$ is accompanied with the formation of the pairs of vortices and antivortices. Concerning the specific heat, a position of a tiny peak superimposed on the left side of a round maximum, corresponds to the maximum of the temperature derivative of the vortex density, while a phase transition of Berezinskii-Kosterlitz-Thouless type is set at lower temperature. For $\Delta_{\lambda}=2 \times 10^{-2}$, the uniform susceptibility follows the behavior of the HAF on the square lattice down to 0.4J. At lower temperatures, the transverse and longitudinal susceptibility separate from the isotropic Heisenberg curve well above the transition temperature, $T_{\mathrm{BKT}}$; at $T_{\mathrm{CO}}$, the $\chi^{\mathrm{zz}}$ component displays a minimum, while $\chi^{\mathrm{xx}}$ decreases faster than the isotropic Heisenberg curve, achieving some nonzero value at $T=0$. As authors showed, in the experiments with the real quasi-2D quantum magnets, the measurements of a single-crystal uniform susceptibility can help to determine the onset of a phase transition as well as the type of the spin anisotropy. If a minimum in the $\chi^{\mathrm{zz}}$ component is observed above $T_{\mathrm{N}}$ (i.e., the temperature of a phase transition to the 3D LRO), this is a signature of the easy-plane anisotropy, while the occurrence of the minimum at the transition temperature suggests the easy-axis anisotropy (Figure 10a).

The QMC studies [97] revealed that the critical temperatures $T_{\mathrm{I}, \mathrm{BKT}}$ remain finite for any finite easy-plane and easy-axis anisotropy (Figure 10b). Thus, unlike the isotropic Heisenberg model on the 
square lattice, for the $\mathrm{XXZ}$ analogue, the quantum and thermal fluctuations are not able to destroy the phase transitions at finite temperatures.

The compound $\mathrm{Sr}_{2} \mathrm{CuO}_{2} \mathrm{Cl}_{2}$ was identified as the first experimental $S=1 / 2 \mathrm{XXZ}$ square-lattice antiferromagnet with a huge intra-layer coupling $J / k_{\mathrm{B}}=1450 \mathrm{~K}$, extremely weak inter-layer coupling $J^{\prime \prime} / J \cong 10^{-5}$ and extremely weak easy-plane anisotropy $\Delta_{\lambda} \approx 10^{-3}[96]$.

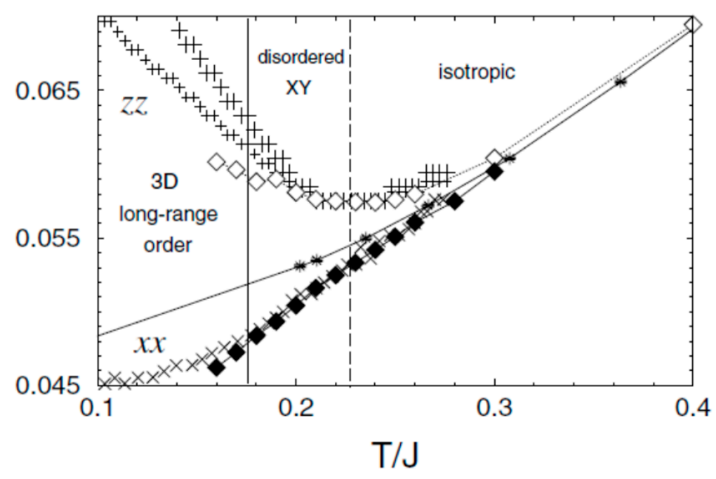

(a)

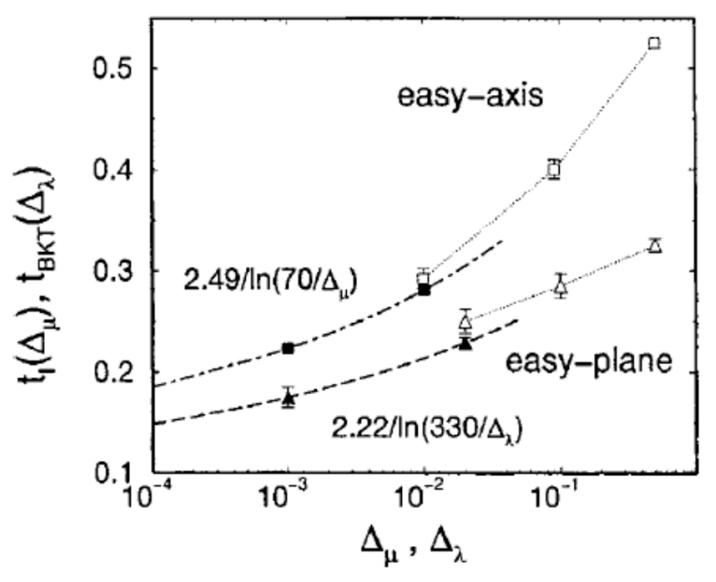

(b)

Figure 10. (a) Uniform susceptibility of $\mathrm{Sr}_{2} \mathrm{CuO}_{2} \mathrm{Cl}_{2}$ (thin and thick pluses represent $\chi^{z z}$, crosses - $\chi^{x x}$ ) compared to the theoretical predictions for the $S=1 / 2$ easy-plane XXZ model on the square lattice with $\Delta_{\lambda}=10^{-3}$ ( $\chi^{z z}$-open diamonds, $\chi^{x x}$-full diamonds). Stars represent the susceptibility of the isotropic HAF on the square lattice. (Reproduced with permission from reference [96]); (b) Phase diagram of the $S=1 / 2 \mathrm{XXZ}$ model on the square lattice for weak anisotropies. The curves in the low-anisotropy region, i.e., $\Delta_{\mu, \lambda}<10^{-1}$, represent the behavior of the reduced critical temperatures $t_{I, B K T}=k_{B} T_{I, B K T} / J$, described by the corresponding expressions. (Reproduced with permission from reference [97]).

A careful analysis of the low-temperature single-crystal susceptibility and magnetization of other $\mathrm{Cu}$ (II) based quantum magnets, $\left.\mathrm{Cu}(\mathrm{pz})_{2}\left(\mathrm{NO}_{3}\right)\right]\left[\mathrm{PF}_{6}\right], \mathrm{Cu}(\mathrm{pz})_{2}\left(\mathrm{ClO}_{4}\right)_{2}$, and $\mathrm{Cu}(\mathrm{pz})_{2}\left(\mathrm{BF}_{4}\right)_{2}$, allowed to determine the presence of the weak easy-plane anisotropy $\Delta_{\lambda} \approx 10^{-3}$ [59]. In these quasi-2D $S=1 / 2 \mathrm{XXZ}$ square-lattice magnets, the intra-layer coupling is two orders of magnitude lower than in $\mathrm{Sr}_{2} \mathrm{CuO}_{2} \mathrm{Cl}_{2}$. The authors found that for the compounds with the high degree of the lattice two-dimensionality (i.e., $J^{\prime \prime} / J \leq 10^{-3}$ ), the spin anisotropy correlates well with the ratio of the anisotropy field, $B_{A}$, and the saturation field, $\Delta_{\lambda} \approx B_{A} / B_{\text {sat }}$ while a strong inter-layer coupling disturbs this coincidence [59].

\section{2. $B \neq 0$}

Extensive theoretical studies of the antiferromagnetic XXZ model on the square lattice in the external magnetic field revealed, that depending on the field orientation, a crossover between different spin symmetries can be induced. The studies of the classical as well as quantum version of the easy-plane XXZ model (Equation (8) with $\Delta_{\mu}=0,0<\Delta_{\lambda} \leq 1$ ) showed the reinforcement of the XY anisotropy when the field is applied along the hard axis $z[98,99]$. On the other hand, at least in the classical version of the XXZ model with the strong easy-plane anisotropy, the application of the magnetic field within the easy plane $(B \perp z)$ breaks the $X Y$ symmetry, introducing the symmetry of Ising type and a transition to the 2D LRO can be expected [98]. However, for the extremely small easy-plane anisotropy, the behavior of the AF XXZ model on the square lattice in $B \perp z$ is unclear.

In the case of the easy-axis XXZ model (Equation (8) with $\Delta_{\lambda}=0,0<\Delta_{\mu} \leq 1$ ) in the magnetic field perpendicular to the easy $z$ axis, $B \perp z$, the system retains a weak Ising anisotropy at all fields up to the saturation value. The ordered AF phase is separated from the paramagnetic phase by a line of the second-order transitions within the 2D Ising universality class [100]. The magnetic field 
applied along the easy axis $(B \| z)$ competes with the spin anisotropy which tends to align the spins along the easy axis. Classical Monte Carlo studies of this 2D model showed the persistence of the collinear Néel order in low magnetic fields. The ordered antiferromagnetic phase is separated from the paramagnetic phase by a line of second-order phase transitions within the 2D Ising universality class [101-104]. The magnetic field acts as the effective easy-plane anisotropy, thus further increase leads to the spin-flop (SF) transition at the field, $B_{\mathrm{SF}}$, accompanied with the reorientation of spins to be orthogonal to the field and gradually canting in its direction. The spin-flop phase is separated from the paramagnetic one by a critical line of BKT transitions [101-104]. In the limit $\Delta_{\mu} \rightarrow 0$, the ordered AF phase gradually vanishes, i.e., $B_{\mathrm{SF}} \rightarrow 0$ and in the isotropic HAF limit, only the spin flop phase remains, separated from the paramagnetic state by the critical line of BKT transitions [101,105].

Quantum Monte Carlo studies of the $S=1 / 2 \mathrm{HAF}$ on the square lattice in the external magnetic field described by the Hamiltonian

$$
H=H_{0}-g \mu_{B} B \sum_{i} S_{i}^{z}
$$

( $H_{0}$ is the Hamiltonian described by the Equation (8) for $\Delta_{\mu}=\Delta_{\lambda}=0$ and $i=\left(i_{1}, i_{2}\right)$ runs over the sites of the square lattice), were performed in a wide range of magnetic fields $h=g \mu_{B} B /(S J)$ from zero to the saturation field $h_{\text {sat }}=8[106,107]$. It was found, that alike in the classical counterpart [101,105], the infinitesimal uniform field induces a BKT transition at a finite temperature. The magnetic phase diagram is characterized by a non-monotonous behavior of the critical temperatures (Figure 11a). In the weak field, the Hamiltonian (Equation (10)) can be mapped on the easy-plane XXZ model in zero magnetic field (Equation (8)) with $\Delta_{\lambda} \approx 0.1 h^{2}$ and the transition temperature

$$
\frac{k_{B} T_{B K T}}{J} \cong \frac{4 \pi \rho_{s} / J}{\ln \left(C / h^{2}\right)}
$$

In higher fields, two competing effects appear; a suppression of the fluctuations of the $S^{z}$ component, resulting in the enhancement of the effective easy-plane anisotropy, which tends to increase $T_{\mathrm{BKT}}$.

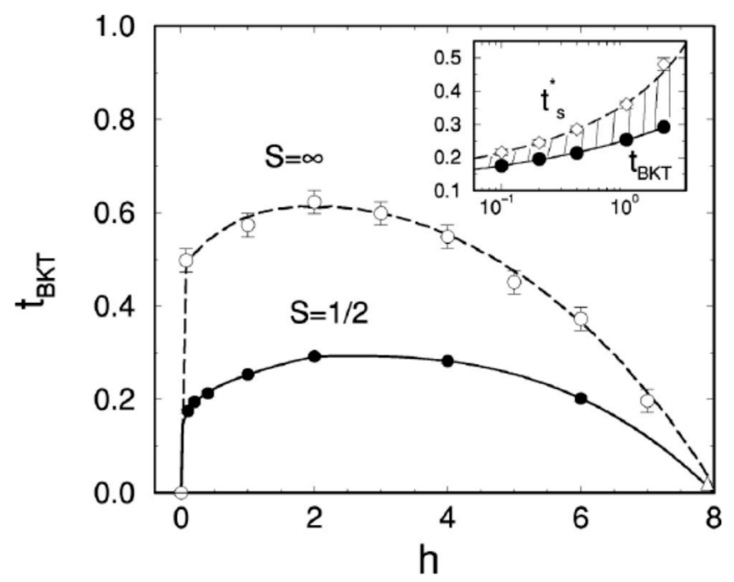

(a)

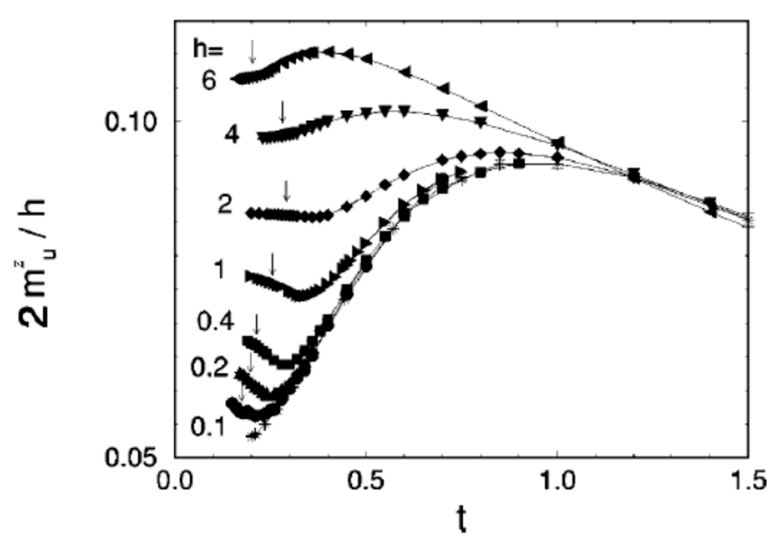

(b)

Figure 11. (a) Magnetic phase diagram of the $S=1 / 2$ HAF on the square lattice (full symbols) in reduced coordinates $t_{B K T}=k_{B} T_{B K T} / J$ and $h=g \mu_{B} B /(S J)$. Open symbols refer to the classical limit of the model. Inset: $t_{B K T}$ vs. $h$ for weak fields, $t_{s}^{*}$ represents a crossover temperature from the isotropic to $X Y$ behavior. Shaded area marks the region of disordered XY behavior. (Reproduced with permission from reference [106]); (b) Field-induced uniform magnetization vs reduced temperature for different field values. The stars represent the zero-field uniform susceptibility of the $S=1 / 2 \mathrm{HAF}$ on the square lattice. The arrows indicate the onset of BKT transition. (Reproduced with permission from reference [106]). 
On the other hand, the average projection of the spins in the $x y$ plane decreases, the system behaves as a planar rotator with a reduced rotator length, which leads to the reduction of $T_{\mathrm{BKT}}$. The effective rotator length goes to zero at the saturation field and corresponding BKT critical temperature vanishes. Apparently, the interplay between the two effects is responsible for the non-monotonous dependence of $T_{\mathrm{BKT}}$, which for the $S=1 / 2$ achieves maximum values at higher fields than in the classical counterpart (Figure 11a). This shift was ascribed to the effect of quantum fluctuations [106].

The QMC simulations of the finite-temperature properties of the $S=1 / 2 \mathrm{HAF}$ on the square lattice (Equation (10)) including the specific heat and uniform magnetization revealed typical features associated with the onset of the XY regime above the BKT transition. In the specific heat, the onset of the BKT transition demonstrates at the temperatures about $20-30 \%$ below a field-induced narrow peak superimposed on the round maximum. The peak is associated with a massive unbinding pairs of vortices (V) and antivortices (AV). A comparative study of the entropy derivatives in $h=0$ and $h>0$ revealed, that for $T<T_{\mathrm{BKT}}$, the entropy in $h>0$ grows slower due to the presence of the quasi-LRO established in the magnetic field after binding $\mathrm{V}+\mathrm{AV}$ pairs, while above $T_{\mathrm{BKT}}$, the growth is much faster due to unbinding $\mathrm{V}+\mathrm{AV}$ pairs. Finally, at high temperatures, the entropy growth does not depend on the field in fully disordered systems [106]. A uniform magnetization is another bulk quantity, which can be experimentally measured; the QMC calculations showed that for $h<1$, the temperature dependence of the uniform magnetization coincides with the uniform susceptibility of the $S=1 / 2 \mathrm{HAF}$ on the square lattice in $h=0$. Deviations appear at low temperatures $k_{\mathrm{B}} T<J$, displaying a minimum, which indicates a field-induced crossover from the isotropic to the $X Y$ regime (Figure 11b).

In the real quasi-two-dimensional magnets, the strength of the interlayer coupling determines to which extent the aforementioned features characterizing the ideal 2D systems can be preserved. Depending on the type of the spin anisotropy and orientation of magnetic field, a corresponding 3D critical behavior can change from the already mentioned 3D Heisenberg to the 3D XY or 3D Ising universality class.

Recent experimental studies of real $S=1 / 2$ quantum magnets with the extremely weak inter-layer coupling revealed a non-monotonous behavior of the 3D transition temperature in the magnetic field analogic to the $B-T$ diagram in Figure 11a. Quantum Monte Carlo studies of the $S=1 / 2$ HAF on the spatially anisotropic simple cubic lattice (with $R=1$ within layers) in the magnetic field [108] simulated the experimental specific heat of the quasi-two-dimensional $S=1 / 2$ quantum antiferromagnet $\left[\mathrm{Cu}\left(\mathrm{HF}_{2}\right)(\mathrm{pz})_{2}\right] \mathrm{BF}_{4}$ with $J / k_{\mathrm{B}}=5.9 \mathrm{~K}$ and $J^{\prime \prime} / J \approx 3 \times 10^{-2}$. In zero magnetic field, the compound undergoes a phase transition to the $3 \mathrm{D} \mathrm{LRO}$ at $T_{\mathrm{N}}=1.6 \mathrm{~K}$. The application of the magnetic field up to $8 \mathrm{~T}$ led to the enhancement of the transition temperature, and a further field increase resulted in the conventional reduction of $T_{\mathrm{N}}(B)$. While in the presence of the nonzero $J$ " the finite-temperature critical 2D properties are lost, the QMC studies found that in the quasi-2D magnets with extremely weak inter-layer coupling, this is just the nonmonotonic behavior of $T_{N}(B)$, which preserves also in the real systems (Figure 12a). On the other hand, the strong $J^{\prime \prime}$ will smear even this feature characterizing the ideal 2D magnets and a conventional decrease of the transition temperature will be observed in all magnetic fields. Such behavior was observed in $(5 \mathrm{CAP})_{2} \mathrm{CuCl}_{4}$, the quasi-2D $S=1 / 2 \mathrm{HAF}$ on the square lattice with $J^{\prime \prime} / J \approx 0.25$ [64].

While extremely weak inter-layer coupling in $\mathrm{Cu}(\mathrm{tn}) \mathrm{Cl}_{2}$ did not allow the formation of a sharp specific heat $\lambda$-like anomaly in the zero magnetic field, the application of the magnetic field of $0.75 \mathrm{~T}$ was capable to induce a weak anomaly at about $0.7 \mathrm{~K}$. A further increase of the field enhanced the anomaly, shifting its position towards higher temperatures. In the fields above $2 \mathrm{~T}$, the amplitude gradually decreased and the anomaly shifted to lower temperatures [89].

A typical increase of $T_{\mathrm{N}}(B)$ was also observed in the $\mathrm{Cu}(\mathrm{pz})_{2}\left(\mathrm{ClO}_{4}\right)_{2}$ with $J^{\prime \prime} / J<10^{-3}$. Since the saturation field is very large, the $B$ - $T$ phase diagram was recorded only for the fields lower than $B_{\text {sat }} / 4$. This value corresponds to the fields below which, the BKT temperature in the ideal 2D case of the $S=1 / 2 \mathrm{HAF}$ on the square lattice grows with the magnetic field [109]. The fact, that the phase 
diagrams of the $\mathrm{Cu}(\mathrm{pz})_{2}\left(\mathrm{ClO}_{4}\right)_{2}$ measured in the fields parallel and perpendicular to magnetic layers were found to be identical, was ascribed to a very weak intrinsic spin anisotropy.

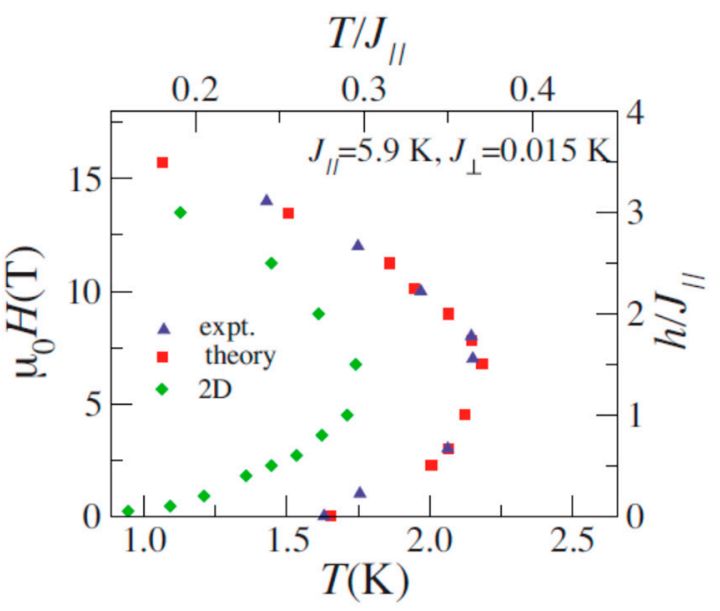

(a)

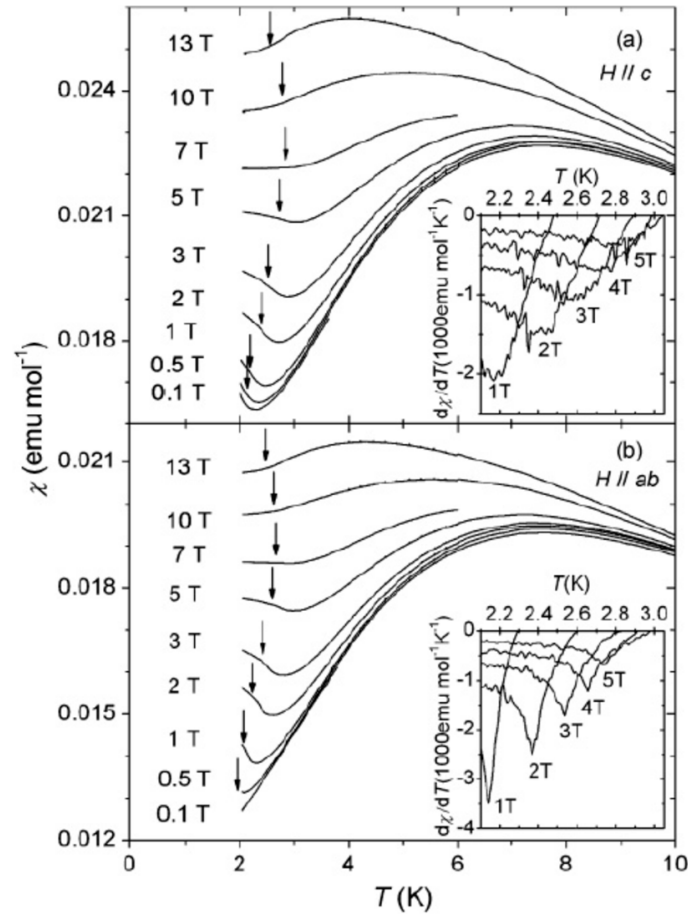

(b)

Figure 12. (a) Magnetic phase diagram of $\left[\mathrm{Cu}\left(\mathrm{HF}_{2}\right)(\mathrm{pz})_{2}\right] \mathrm{BF}_{4}$ compared with $\mathrm{QMC}$ simulations of the $S=1 / 2$ HAF on the spatially anisotropic simple cubic lattice $(R=1$ within layers) with the system parameters $\left(J_{\|} \equiv J_{,} J_{\perp} \equiv J^{\prime \prime}, h=g \mu_{B} \mu_{0} H\right)$. For comparison, the results for the pure 2D case $\left(J^{\prime \prime}=0\right)$ are also shown. (Reproduced with permission from reference [108]); (b) $\chi(T)$ of $\left[\mathrm{Cu}(\mathrm{pz})_{2}(\mathrm{pyO})_{2}\right]\left(\mathrm{PF}_{6}\right)_{2}$ for the magnetic field applied perpendicular to the easy plane $a b\left(\mu_{0} H \| c\right)$ and within the easy plane $a b$ $\left(\mu_{0} H \| a b\right)$. The arrows mark the positions of the specific heat peaks. The insets show $\partial \chi(T) / \partial T$ below 5 T. (Reproduced with permission from reference [90]).

As for the case of $\mathrm{Cu}(\mathrm{tn}) \mathrm{Cl}_{2}$, no sharp specific heat anomaly was observed down to $0.5 \mathrm{~K}$ in $\left[\mathrm{Cu}(\mathrm{pz})_{2}(\mathrm{pyO})_{2}\right]\left(\mathrm{PF}_{6}\right)_{2},(\mathrm{pyO}=$ pyridine-N-oxide $)$, a quasi-2D $S=1 / 2 \mathrm{HAF}$ on the square lattice with $J / k_{\mathrm{B}}=8.2 \mathrm{~K}, J^{\prime \prime} / J \approx 2 \times 10^{-4}$ and the weak easy-plane anisotropy $\Delta_{\lambda} \approx 0.007$ [90]. The application of the magnetic field of $1 \mathrm{~T}$ induced a weak anomaly at about $2 \mathrm{~K}$, gradually growing and shifting towards higher temperatures. In relatively low fields, the shape of the anomaly depended on the orientation of the magnetic field. When the field was applied perpendicular to the easy plane, in accord with the theory [99], the field plays a role of an effective easy-plane anisotropy, thus fingerprints of the BKT transition in the whole field region up to $B_{\text {sat }}$ can be expected. Considering the field applied within the easy plane, authors expected a field-induced easy-axis anisotropy, established in the fields up to $5 \mathrm{~T}$ [90]. In these fields, the formation of tiny $\lambda$-like anomalies, typical for the Ising transitions was observed, while at the fields above $5 \mathrm{~T}$, the anomalies evolved to a broad anomaly of the expected BKT type and the system behaved as the isotropic 2D HAF in the field. Authors studied also a temperature dependence of the susceptibility in constant magnetic fields. In both orientations, the susceptibility is characterized by a broad maximum typical for 2D magnets with an upturn below $3 \mathrm{~K}$ (Figure 12b). In the field perpendicular to the easy plane, a minimum occurs always above the transition temperature to the 3D LRO, a feature typical for the onset of the XY regime, while in the field applied within the easy plane, such behavior occurs only above $1 \mathrm{~T}$. Experimental studies of other quasi-2D $S=1 / 2$ antiferromagnets with a weak easy-plane anisotropy in the magnetic field applied within the easy plane found, that for the fields lower than the anisotropy field $B_{\mathrm{A}}$, the temperature dependence of 
the susceptibility has no upturn and its qualitative behavior follows $\chi^{x x}$ in Figure 10a. On the other hand, in the fields exceeding $B_{\mathrm{A}}$, the upturn gradually develops and a characteristic minimum forms at temperatures above $T_{N}(B)$ as a typical sign of the onset of the field-induced $X Y$ regime (see Figure 8 of reference [59]). Thus, considering an excellent spatial two-dimensionality of $\left[\mathrm{Cu}(\mathrm{pz})_{2}(\mathrm{pyO})_{2}\right]\left(\mathrm{PF}_{6}\right)_{2}$, the application of the aforementioned relation $\Delta_{\lambda} \approx B_{A} / B_{\text {sat }}$ provides $B_{\mathrm{A}} \approx 0.2 \mathrm{~T}$. Taking into account a combined effect of the inter-layer coupling and the spin anisotropy, some higher external field should compensate both effects, to set the 2D XY regime. The experimental susceptibility data in the fields applied within the easy plane in Figure $12 \mathrm{~b}$ suggest, that this condition has already been fulfilled at least for $B=1 \mathrm{~T}$.

It should be noted, that at present, there are no theoretical studies of the $S=1 / 2 \mathrm{XXZ}$ model with the extremely weak easy-plane anisotropy in the magnetic field applied within the easy plane which would provide reliable information about the field-induced spin crossover between the Ising and the $X Y$ regime. Despite the absence of the theoretical work, experimental studies of the magnetic phase diagrams of the spin $1 / 2$ quasi-2D magnets were performed, to investigate the field-induced spin crossover in detail. Previous studies of $\mathrm{Cu}(\mathrm{pz})_{2}\left(\mathrm{ClO}_{4}\right)_{2}$, the $2 \mathrm{D}$ square-lattice magnet with $J^{\prime \prime} / J \approx 8.8 \times 10^{-4}, T_{\mathrm{N}}=4.2 \mathrm{~K}$ and $B_{\text {sat }} \approx 49 \mathrm{~T}$, identified the presence of the easy-plane anisotropy $\Delta_{\lambda} \approx 4.6 \times 10^{-3}$ [59]. Subsequent antiferromagnetic resonance experiments and magnetization measurements in the ordered phase refined the character of the spin anisotropy comprising of the out-of-plane (easy-plane) anisotropy $\Delta_{\lambda} \approx 3.1 \times 10^{-3}$ and the in-plane anisotropy $\Delta_{\text {in }} \approx 3.1 \times 10^{-4}$, the latter breaking a continuous symmetry within the easy $x y$ plane $[94,110]$. Thus, the description of $\mathrm{Cu}(\mathrm{pz})_{2}\left(\mathrm{ClO}_{4}\right)_{2}$ within the $\mathrm{XYZ}$ model with extremely weak anisotropy is more realistic.

The interplay of the in-plane anisotropy and magnetic field was already theoretically investigated in the AF chains [16], thus some qualitative conclusions can be extrapolated also to the 2D systems. For the field applied along the easy axis $x$, the magnetic field opposes the effect of the in-plane anisotropy. The resulting effective anisotropy $\Delta_{e f f}=\Delta_{i n}-a\left(g \mu_{B} B / J\right)^{2}$ controls the ground-state symmetry [16]. For the small magnetic field, the intrinsic anisotropy dominates, stabilizing the collinear AF Néel order along the $x$ axis. With the increasing field, the influence of $\Delta_{\text {in }}$ gradually weakens and finally, a spin-flop transition from the easy $(x)$ to the middle axis $(y)$ occurs within the easy plane [16].

In $\mathrm{Cu}(\mathrm{pz})_{2}\left(\mathrm{ClO}_{4}\right)_{2}$, for the field applied along the easy axis, the AF-SF critical line ended in a bicritical point at $4 \mathrm{~K}$ and the field about $0.73 \mathrm{~T}$ (Figure 13). For the constant fields $B<0.5 \mathrm{~T}$, the temperature dependence of the corresponding normalized uniform magnetization $M(T) / B$ is characterized by a sharp change of the slope in the vicinity of the transition temperature, separating the collinear AF Néel phase and the paramagnetic (PM) phase. For the fields, $0.5<B<0.73 \mathrm{~T}$, the $M(T) / B$ curves cross the SF-AF and AF-PM critical lines. The former crossing is accompanied with a pronounced step in the curves (Figure 13a). For higher fields, the spin-flop phase is stabilized with the $X Y$ regime, manifesting by the upturn in the $M(T) / B$ curves with a minimum, ascribed to the $2 \mathrm{D}$ Heisenberg-XY crossover. The kinks in the curves were associated with the onset of the 3D LRO. The application of the field along the hard axis $z$, leads to the behavior of $M(T) / B$ typical for the XY regime, since the magnetic field enforces the effect of the intrinsic easy-plane anisotropy, resulting in the effective easy-plane anisotropy $\Delta_{e f f}=\Delta_{\lambda}+a\left(g \mu_{B} B / J\right)^{2}$ [94]. Concerning the middle axis $y$, authors of reference [16] expected enforcing of the in-plane easy-axis anisotropy, $\Delta_{e f f}=\Delta_{i n}+a\left(g \mu_{B} B / J\right)^{2}$. However, as was shown in $\mathrm{Cu}(\mathrm{pz})_{2}\left(\mathrm{ClO}_{4}\right)_{2}$, in this orientation, the fields above $2 \mathrm{~T}$ introduced the $\mathrm{XY}$ regime with the effective anisotropy $\Delta_{e f f} \approx a\left(g \mu_{B} B / J\right)^{2}$ (Figure 16 in reference [94]). Apparently, alike in the case of the aforementioned easy-plane XXZ model, the theoretical studies of the $S=1 / 22 \mathrm{D}$ $\mathrm{XYZ}$ model on the square lattice with a weak spin anisotropy are necessary, to verify the persistence of the Ising-like ground state as well as the Ising- $X Y$ crossover, induced by the field applied along the middle axis. 

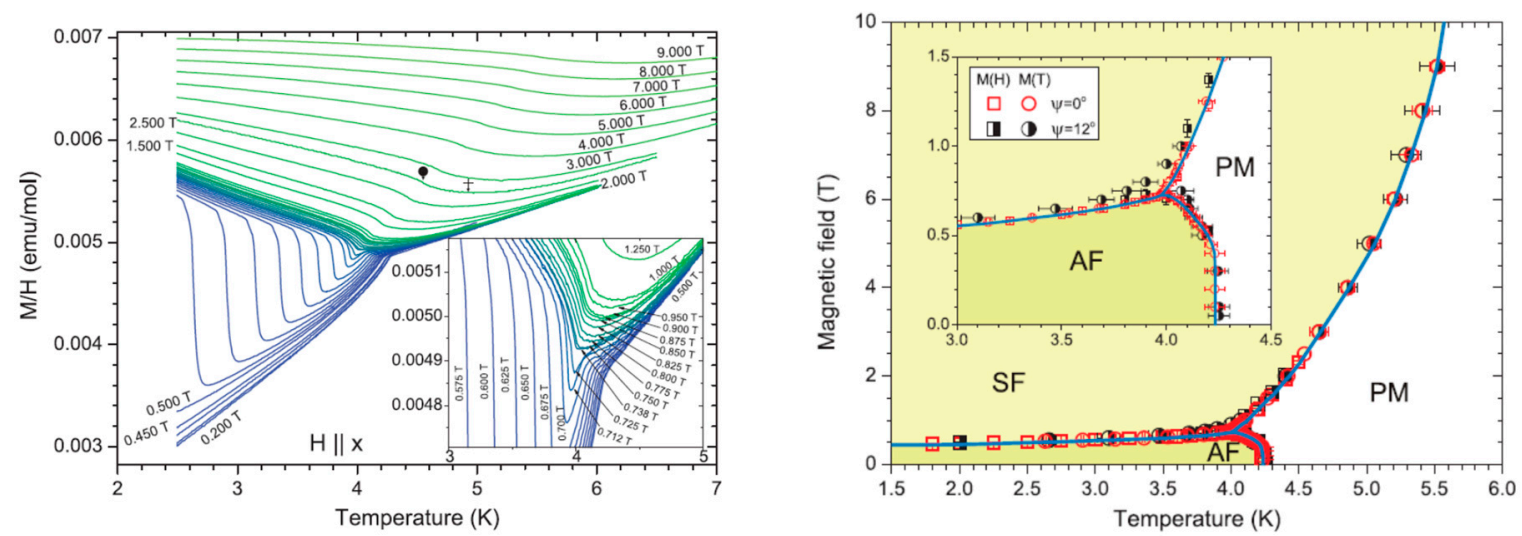

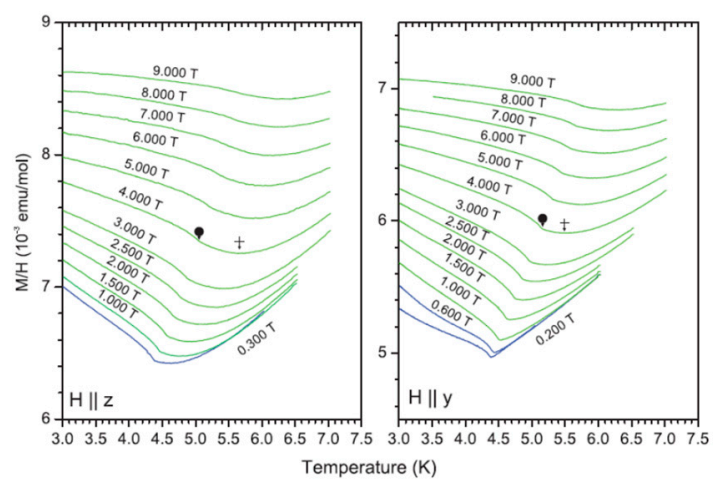

(a)

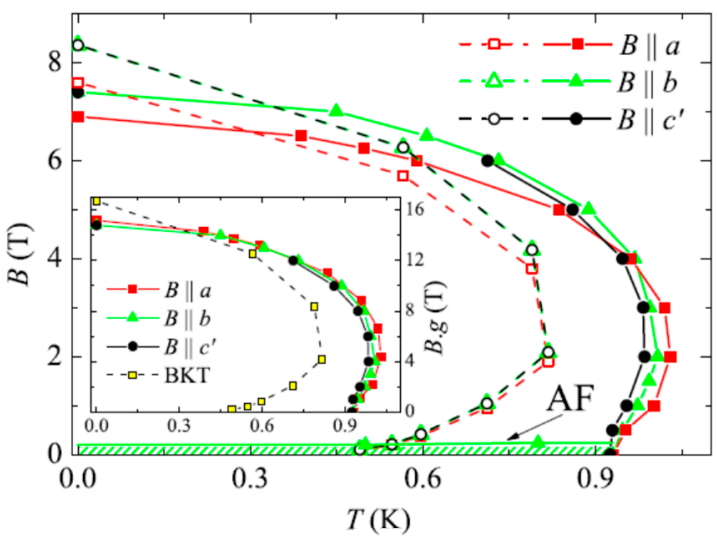

(b)

Figure 13. (a) (top, bottom) Normalized magnetization $M(T) / B$ of $\mathrm{Cu}(\mathrm{pz})_{2}\left(\mathrm{ClO}_{4}\right)_{2}$ for various magnetic fields $B \equiv \mu_{0} H$ applied along the easy $(x)$, middle $(y)$ and hard $(z)$ axis. In all three cases, the cross and droplet symbols mark the minimum and kink, respectively. (Reproduced with permission from reference [94]); (b) (top) Magnetic phase diagram of $\mathrm{Cu}(\mathrm{pz})_{2}\left(\mathrm{ClO}_{4}\right)_{2}$ in the field applied along the easy axis. AF, SF and PM denote the collinear antiferromagnetic phase, the spin-flop phase and the paramagnetic phase, respectively. The phase boundaries are constructed from the anomalies in the $M(T)$ and $M(H)$ curves, red and black points correspond to the orientation of the field $\left(\psi=0^{\circ}\right.$ and $12^{\circ}$ ) with respect to the easy axis. Lines are a guide to the eye. In the inset, an expanded region around the bicritical point is shown. (Reproduced with permission from reference [94]); (b) (bottom) Magnetic phase diagram of $\mathrm{Cu}(\mathrm{en})\left(\mathrm{H}_{2} \mathrm{O}\right)_{2} \mathrm{SO}_{4}$. The $a, b, c^{\prime}$ axes correspond to the hard, easy and middle axis, respectively. Open symbols represent the theoretical predictions [97] for a field-induced BKT transition in the $S=1 / 2 \mathrm{HAF}$ square lattice $\left(R=1, J / k_{B} \equiv J_{e f f} / k_{B}=2.8 \mathrm{~K}, g_{a}=2.200, g_{b}=2.005\right.$ and $g_{c^{\prime}}=2.000$ ). Inset: the same diagram, $B$ is replaced by the product $B \cdot g$. (Reproduced with permission from reference [82]).

The analysis of thermodynamic properties showed that also $\mathrm{Cu}(\mathrm{en})\left(\mathrm{H}_{2} \mathrm{O}\right)_{2} \mathrm{SO}_{4}$ can be treated as the realization of the $S=1 / 22 \mathrm{D} \mathrm{XYZ} \mathrm{model} \mathrm{[82].} \mathrm{In} \mathrm{this} \mathrm{respect,} \mathrm{both,} \mathrm{Cu}(\mathrm{pz})_{2}\left(\mathrm{ClO}_{4}\right)_{2}$ and $\mathrm{Cu}(\mathrm{en})\left(\mathrm{H}_{2} \mathrm{O}\right)_{2} \mathrm{SO}_{4}$ should represent the same model, i.e., the $S=1 / 2 \mathrm{XYZ}$ model on the spatially anisotropic zig-zag square lattice, differing in the amount of the spatial and the spin anisotropy. Within the effective $S=1 / 2 \mathrm{HAF}$ square lattice model, the rescaling $B_{\text {sat }} / 7$ and $J_{\text {eff }} /\left(7 k_{\mathrm{B}}\right)$ parameters of $\mathrm{Cu}(\mathrm{pz})_{2}\left(\mathrm{ClO}_{4}\right)_{2}$ will provide $B_{s a t}^{*} \approx 7 \mathrm{~T}$ and $J_{e f f}^{*} / k_{B} \approx 2.6 \mathrm{~K}$, the values which are reported for $\mathrm{Cu}(\mathrm{en})\left(\mathrm{H}_{2} \mathrm{O}\right)_{2} \mathrm{SO}_{4}$ [65]. Concerning the spin anisotropy, applying the relation for the anisotropy-induced energy gaps in the excitation spectrum [94]

$$
E_{g}^{\text {in,out }} \cong 1.2 \sqrt{2 J\left(\Delta J^{\text {in,out }}\right)}
$$


for the rescaled $J_{e f f}^{*} / k_{B}$ and the gap value $E_{g}^{i n} \approx 0.3 \mathrm{~K}$ reported for $\mathrm{Cu}(\mathrm{en})\left(\mathrm{H}_{2} \mathrm{O}\right)_{2} \mathrm{SO}_{4}$ [82], the relation provides the in-plane anisotropy $\Delta J^{i n} \approx 12 \mathrm{mK}$, which is about two times larger than $\Delta J^{i n} \approx 5.3 \mathrm{mK}$ reported for $\mathrm{Cu}(\mathrm{pz})_{2}\left(\mathrm{ClO}_{4}\right)_{2}$ [94]. This result correlates well with the recent antiferromagnetic resonance experiments in $\mathrm{Cu}(\mathrm{en})\left(\mathrm{H}_{2} \mathrm{O}\right)_{2} \mathrm{SO}_{4}$ [111]. In addition, they determined the ratio of the spin gaps $E_{g}^{\text {out }} / E_{g}^{i n} \approx 2.3$, correspondingly $\Delta J^{\text {out }} / \Delta J^{i n} \approx 5$. In $\mathrm{Cu}(\mathrm{pz})_{2}\left(\mathrm{ClO}_{4}\right)_{2}$, the ratio of the spin gaps and the spin anisotropies is about 3.3 and 10, respectively, which is much closer to the easy-plane XXZ model. Apparently, the size as well as the relative strength of the in-plane anisotropy in $\mathrm{Cu}(\mathrm{en})\left(\mathrm{H}_{2} \mathrm{O}\right)_{2} \mathrm{SO}_{4}$ is two times larger than in $\mathrm{Cu}(\mathrm{pz})_{2}\left(\mathrm{ClO}_{4}\right)_{2}$. It seems, that this difference correlates well with the difference in the spatial anisotropies; $R=0.7 \mathrm{in} \mathrm{Cu}(\mathrm{pz})_{2}\left(\mathrm{ClO}_{4}\right)_{2}$ is two times lower than $R=0.35$ in $\mathrm{Cu}(\mathrm{en})\left(\mathrm{H}_{2} \mathrm{O}\right)_{2} \mathrm{SO}_{4}$.

Previous studies of the electron paramagnetic resonance spectra in $\mathrm{Cu}(\mathrm{en})\left(\mathrm{H}_{2} \mathrm{O}\right)_{2} \mathrm{SO}_{4}$ already revealed the aforementioned strong rhombic character of the spin anisotropies [80]. It was assumed, that a dipolar coupling between nearest neighbors could provide the main source of the spin anisotropy in $\mathrm{Cu}(\mathrm{en})\left(\mathrm{H}_{2} \mathrm{O}\right)_{2} \mathrm{SO}_{4}$, where the exchange pathways are very long, resulting in weak exchange couplings. Consequently, the spin anisotropy could reflect the spatial distribution of $\mathrm{Cu}$ (II) magnetic moments. The dipolar coupling was considered as the only origin of the extremely weak easy-axis anisotropy $\Delta_{\mu} \approx 10^{-4}$, observed in the verdazyl radical [85]. As for $\mathrm{Cu}(\mathrm{pz})_{2}\left(\mathrm{ClO}_{4}\right)_{2}$, the exchange coupling is much stronger than in $\mathrm{Cu}(\mathrm{en})\left(\mathrm{H}_{2} \mathrm{O}\right)_{2} \mathrm{SO}_{4}$, but the strength of the in-plane anisotropy is weaker than in $\mathrm{Cu}(\mathrm{en})\left(\mathrm{H}_{2} \mathrm{O}\right)_{2} \mathrm{SO}_{4}$. The origin of the in-plane spin anisotropy in $\mathrm{Cu}(\mathrm{pz})_{2}\left(\mathrm{ClO}_{4}\right)_{2}$ was ascribed to the weak rhombic distortion of the crystal lattice, being of the same order as the spin anisotropy [94]. Similar symmetry arguments were used to explain the extremely weak spin anisotropy in $\left[\mathrm{Cu}\left(\mathrm{HF}_{2}\right)(\mathrm{pz})_{2}\right] \mathrm{SbF}_{6}$ with a tetragonal structural symmetry in the ordered phase, while stronger anisotropies observed in the $\mathrm{Cu}$ (II) magnets with lower crystal symmetry were ascribed to their reduced structural symmetry [84].

Experimental studies of the $\mathrm{Cu}(\mathrm{II})$ compounds showed that sufficiently large magnetic fields, $g \mu_{B} B>E_{g}^{i n, o u t}$, can overcome the effect of the intrinsic spin anisotropies and the systems behave as a Heisenberg antiferromagnet in the magnetic field, which induces the $\mathrm{XY}$ regime [82,94]. As was demonstrated in $\mathrm{Cu}(\mathrm{en})\left(\mathrm{H}_{2} \mathrm{O}\right)_{2} \mathrm{SO}_{4}$, after rescaling magnetic field with the high-temperature $g$-factors (to remove the effects of the local surrounding of $\mathrm{Cu}$ (II) ions introduced via a spin-orbit coupling), the magnetic fields exceeding the $B_{\mathrm{SF}}$ values, produce in all orientations the same behavior of the transition temperatures (Figure 13b). A comparison of the rescaled magnetic diagram with that for the $S=1 / 2$ 2D HAF model on the square lattice $(R=1)$ revealed large discrepancies for $B \approx 0$, due to the presence of the inter-layer coupling in $\mathrm{Cu}(\mathrm{en})\left(\mathrm{H}_{2} \mathrm{O}\right)_{2} \mathrm{SO}_{4}$. On the other hand, the differences in the vicinity of the saturation field result from the higher measure of the quantum fluctuations in $\mathrm{Cu}(\mathrm{en})\left(\mathrm{H}_{2} \mathrm{O}\right)_{2} \mathrm{SO}_{4}$, introduced by the spatial anisotropy of the intra-layer exchange coupling.

\section{Summary and Concluding Remarks}

The effects of the spatial anisotropy of the exchange coupling as well as the intrinsic or magnetic-field induced spin anisotropy were discussed in the two-dimensional magnetic models and their experimental counterparts realized by the low-dimensional $\mathrm{Cu}(\mathrm{II})$ based metal-organic magnets.

The ground-state and finite-temperature properties of the $S=1 / 2 \mathrm{HAF}$ chain model and HAF on the square lattice with the nearest neighbors were presented separately as important paradigms of the low-dimensional magnetism. Then, the attention was focused on the $S=1 / 2$ HAF model on the rectangular lattice, which interpolates between the aforementioned 1D and 2D models, enabling the tuning the strength of the quantum fluctuations, which destabilize the ordered collinear Néel ground state when approaching the 1D limit. The problems with the distinction between the square, rectangular, and zig-zag square lattice in the analysis of the experimental data are discussed and the importance of the specific heat data and first-principle calculations is demonstrated on individual examples. 
The impact of the inter-layer exchange coupling on the stabilization of the 3D long-range order in the quasi-2D magnets was presented. An ambiguous indication of the phase transition in the experimental data can occur when the inter-layer coupling is too weak. Besides neutron scattering, other experimental methods, as antiferromagnetic resonance or magnetic susceptibility can provide a proper information on the onset of the 3D LRO.

The effect of the extremely weak spin anisotropy in the 2D systems was presented by the $S=1 / 2$ easy-axis and easy-plane XXZ models on the square lattice in zero and nonzero magnetic field. In $B=0$, theoretical studies predict the onset of the 2D LRO, induced by the easy-axis anisotropy, while Berezinskii-Kosterlitz-Thouless phase transition appears in the presence of the intrinsic easy-plane anisotropy. It was shown, that under some conditions, the external magnetic field can act as a source of the effective easy-plane anisotropy. The fingerprints of the BKT transition in the real quasi-2D Cu(II) based molecular magnets were discussed. Finally, the interplay of the intrinsic spin anisotropy, magnetic field, and the inter-layer coupling in real quasi-2D $\mathrm{Cu}(\mathrm{II})$ based molecular magnets was demonstrated as specific features of magnetic properties and the corresponding magnetic phase diagrams.

The aforementioned discussion of the 2D theoretical models was restricted to the rectangular lattice with the nearest neighbors. Apparently, even this simple un-frustrated model can provide a rich variety of the phenomena, some of which still waiting for the theoretical and experimental investigations. While the excitation spectra of the $S=1 / 2 \mathrm{HAF}$ chain and the square lattice were studied in detail theoretically and experimentally, analogical studies for $R<1$ are still missing. Concerning the excitation spectrum of the HAF on the square lattice, the formation of a field-induced low-energy roton-like structure with the softening of the roton gap near $B_{\text {sat }}$, was theoretically predicted along with the suggestions, how to observe this feature experimentally [112]. Theoretical studies also predict the existence of the roton minimum in the high-energy part of the excitation spectrum of the same model in zero magnetic field [113].

As was shown in reference [72,74], in the $S=1 / 2 \mathrm{HAF}$ on the rectangular lattice the amount of quantum fluctuations increases when approaching 1D limit. Corresponding theoretical studies of the $S=1 / 2 \mathrm{XXZ}$ model on the rectangular lattice would be desirable to find, to which extent the interplay of the intrinsic/effective spin anisotropy and the enhanced quantum fluctuations can change the physical picture already derived for the XXZ model on the square lattice. Experimental investigations of the spin anisotropy in various quasi-2D quantum magnets found correlations between the character of the spin anisotropy and the structural symmetry. To achieve a 2D Heisenberg limit by minimizing the intra-layer spin anisotropies, the synthesis of molecule-based materials with a large inter-layer spacing and the tetragonal crystal symmetry in the ordered magnetic phase is required [84]. The engineering of proper experimental systems would enable to control the third lattice dimension in the square-lattice antiferromagnets as well as the intra-layer spin anisotropy, to obtain experimental model systems. Besides the chemical synthesis, the application of the pressure represents another tool for the structure modifications of molecular-based magnets. In these materials, hydrogen bonds and other electrostatic contacts play an important role in the packing to the 3D crystal structure with rather soft mechanical properties, characterized by low Debye temperatures and low-energy vibrational modes [114-116]. Correspondingly, unlike inorganic materials, the application of pressure to such soft structures could introduce new properties at relatively low pressures [117,118].

Besides bulk materials, the modification of their properties by a deposition of molecular-based magnets on substrates [119,120], growing in the confined geometry [121], etc. opens new possibilities for the experimental and theoretical investigations. Such studies have already strongly multidisciplinary character, requiring the cooperation of the experts from different fields and besides fundamental research, the results can have potential practical applications e.g., in the information technologies.

Author Contributions: Conceptualization, A.O.; Investigation, E.Č. and R.T.; Writing-Original Draft Preparation, A.O.; Writing-Review \& Editing, R.T.; Visualization, V.T.; Funding Acquisition, M.O. and A.F. 
Funding: The work was supported by VEGA Grant No. 1/0269/17 of the Scientific Grant Agency of the Ministry of Education, Science, Research and Sport of the Slovak Republic and the Slovak Academy of Sciences, the Slovak Research and Development Agency Projects No. APVV-14-0078 and No. APVV-14-0073, and the Project No. ITMS 26220120047 of European Regional Development Fund.

Conflicts of Interest: The authors declare no conflict of interest. The funders had no role in the design of the study; in the collection, analyses, or interpretation of data; in the writing of the manuscript, and in the decision to publish the results.

\section{References}

1. Ising, E. Beitrag zur Theorie des Ferromagnetismus. Z. Phys. 1925, 31, 253-258. [CrossRef]

2. Onsager, L. Crystal Statistics. I. A Two-Dimensional Model with an Order-Disorder Transition. Phys. Rev. 1944, 65, 117-149. [CrossRef]

3. Domb, C. On the Theory of Cooperative Phenomena in Crystals. Adv. Phys. 1960, 9, 149-361. [CrossRef]

4. Mermin, N.D.; Wagner, H. Absence of Ferromagnetism or Antiferromagnetism in One- or Two-Dimensional Isotropic Heisenberg Models. Phys. Rev. Lett. 1966, 17, 1133-1136. [CrossRef]

5. Stanley, H.E.; Kaplan, T.A. Possibility of a Phase Transition for the Two-Dimensional Heisenberg Model. Phys. Rev. Lett. 1966, 17, 913-915. [CrossRef]

6. Wegner, F. Spin-Ordering in a Planar Classical Heisenberg Model. Z. Phys. 1967, 206, 465-470. [CrossRef]

7. Stanley, H.E. Dependence of Critical Properties on Dimensionality of Spins. Phys. Rev. Lett. 1968, 20, 589-592. [CrossRef]

8. Moore, M.A. Additional Evidence for a Phase Transition in the Plane-Rotator and Classical Heisenberg Models for Two-Dimensional Lattices. Phys. Rev. Lett. 1968, 23, 861-863. [CrossRef]

9. Berezinskii, V.L. Destruction of Long-range Order in One-dimensional and Two-dimensional Systems having a Continuous Symmetry Group I. Classical Systems. Sov. Phys. JETP 1971, 32, 493-500.

10. Berezinskii, V.L. Destruction of Long-range Order in One-dimensional and Two-dimensional Systems Possessing a Continuous Symmetry Group. II. Quantum Systems. Sov. Phys. JETP 1971, 34, 610-616.

11. Kosterlitz, M.J.; Thouless, D.J. Ordering, Metastability and Phase Transitions in Two-Dimensional Systems. J. Phys. C 1973, 6, 1181-1203. [CrossRef]

12. Minnhagen, P. The Two-Dimensional Coulomb Gas, Vortex Unbinding, and Superfluid-Superconducting Films. Rev. Modern Phys. 1987, 59, 1001-1066. [CrossRef]

13. de Jongh, L.J.; Miedema, A.R. Experiments on Simple Magnetic Model Systems. Adv. Phys. 1974, $23,1-260$. [CrossRef]

14. Müller, G.; Thomas, H.; Beck, H.; Bonner, J.C. Quantum Spin Dynamics of the Antiferromagnetic Linear Chain in Zero and Nonzero Magnetic Field. Phys. Rev. B 1981, 24, 1429-1467. [CrossRef]

15. Mikeska, H.J. Solitons in a One-Dimensional Magnet with an Easy Plane. J. Phys. C 1978, 11, L29-L32. [CrossRef]

16. Mikeska, H.J.; Steiner, M. Solitary Excitations in One-Dimensional Magnets. Adv. Phys. 1991, 40, $191-356$. [CrossRef]

17. Haldane, F.D.M. Continuum Dynamics of the 1-D Heisenberg Antiferromagnet: Identification with the $\mathrm{O}(3)$ Nonlinear Sigma Model. Phys. Lett. A 1983, 93, 464-468. [CrossRef]

18. Haldane, F.D.M. Nonlinear Field Theory of Large-Spin Heisenberg Antiferromagnets: Semiclassically Quantized Solitons of the One-Dimensional Easy-Axis Néel State. Phys. Rev. Lett. 1983, 50, 1153-1156. [CrossRef]

19. Renard, J.P.; Verdaguer, M.; Regnault, L.P.; Erkelens, W.A.C.; Rossat-Mignod, J.; Stirling, W.G. Presumption for a Quantum Energy Gap in the Quasi-One-Dimensional $S=1$ Heisenberg Antiferromagnet $\mathrm{Ni}\left(\mathrm{C}_{2} \mathrm{H}_{8} \mathrm{~N}_{2}\right)_{2} \mathrm{NO}_{2}\left(\mathrm{ClO}_{4}\right)$. Europhys. Lett. 1987, 3, 945-951. [CrossRef]

20. Ma, S.; Broholm, C.; Reich, D.H.; Sternlieb, B.J.; Erwin, R.W. Dominance of Long-Lived Excitations in the Antiferromagnetic Spin-1 Chain NENP. Phys. Rev. Lett. 1992, 69, 3571-3574. [CrossRef]

21. Dagotto, E. Correlated Electrons in High-Temperature Superconductors. Rev. Mod. Phys. 1994, 66, 763-840. [CrossRef]

22. Huse, D.A.; Elser, V. Simple Variational Wave Functions for Two-Dimensional Heisenberg Spin- $\frac{1}{2}$ Antiferromagnets. Phys. Rev. Lett. 1988, 60, 2531-2534. [CrossRef] [PubMed] 
23. Shastry, B.S.; Sutherland, B. Exact Ground-State of a Quantum-Mechanical Antiferromagnet. Physica B \& C 1981, 108, 1069-1070. [CrossRef]

24. Bernhard, B.H.; Canals, B.; Lacroix, C. Green's Function Approach to the Magnetic Properties of the Kagomé Antiferromagnet. Phys. Rev. B 2002, 66, 104424. [CrossRef]

25. Savary, L.; Balents, L. Quantum Spin Liquids: A Review. Rep. Prog. Phys. 2017, 80, 016502. [CrossRef] [PubMed]

26. Scholwöck, U.; Richter, J.; Farnel, D.J.J.; Bishop, R.F. Quantum Magnetism, 1st ed.; Springer: Berlin/Heidelberg, Germany, 2004; ISBN 978-3-540-40066-0.

27. Blundel, S.J.; Pratt, F.L. Organic and Molecular Magnets. J. Phys. Condens. Matter 2004, 16, R771-R828. [CrossRef]

28. Meier, F.; Levy, J.; Loss, D. Quantum Computing with Spin Cluster Qubits. Phys. Rev. Lett. 2003, 90, 047901. [CrossRef]

29. Jenkins, M.D.; Duan, Y.; Diosdado, B.; García-Ripoll, J.J.; Gaita-Ariño, A.; Giménez-Saiz, C.; Alonso, P.J.; Coronado, E.; Luis, F. Coherent Manipulation of Three-Qubit States in a Molecular Single-Ion Magnet. Phys. Rev. B 2017, 95, 064423. [CrossRef]

30. Gaudenzi, R.; Burzurí, E.; Maegawa, S.; van der Zant, H.S.J.; Luis, F. Quantum Landauer Erasure with a Molecular Nanomagnet. Nat. Phys. 2018, 14, 565-568. [CrossRef]

31. Zhang, X.X.; Wei, H.L.; Zhang, Z.Q.; Zhang, L. Anisotropic Magnetocaloric Effect in Nanostructured Magnetic Clusters. Phys. Rev. Lett. 2001, 87, 157203. [CrossRef]

32. Zhitomirski, M.E. Enhanced Magnetocaloric effect in Frustrated Magnets. Phys. Rev. B 2003, 67, 104421. [CrossRef]

33. Trippe, C.; Honecker, A.; Klümper, A.; Ohanyan, V. Exact Calculation of the Magnetocaloric Effect in the spin-1/2 XXZ Chain. Phys. Rev. B 2010, 81, 054402. [CrossRef]

34. Thomas, L.; Lionti, F.; Ballou, R.; Gatteschi, D.; Sessoli, R.; Barbara, B. Macroscopic Quantum Tunnelling of Magnetization in a Single Crystal of Nanomagnets. Nature 1996, 383, 145-147. [CrossRef]

35. Radu, T.; Wilhelm, H.; Yushankhai, V.; Kovrizhin, D.; Coldea, R.; Tylczynski, Z.; Lühmann, T.; Steglich, F. Bose-Einstein Condensation of Magnons in $\mathrm{Cs}_{2} \mathrm{CuCl}_{4}$. Phys. Rev. Lett. 2005, 95, 127202. [CrossRef] [PubMed]

36. Zurek, W.H.; Dorner, U.; Zoller, P. Dynamics of a Quantum Phase Transition. Phys. Rev. Lett. 2005, 95, 105701. [CrossRef] [PubMed]

37. Blanc, N.; Trinh, J.; Dong, L.; Bai, X.; Aczel, A.A.; Mourigal, M.; Balents, L.; Siegrist, T.; Ramirez, A.P. Quantum Criticality among Entangled Spin Chains. Nat. Phys. 2018, 14, 273-276. [CrossRef]

38. Kasahara, Y.; Sugii, K.; Ohnishi, T.; Shimozawa, M.; Yamashita, M.; Kurita, N.; Tanaka, H.; Nasu, J.; Motome, Y.; Shibauchi, T.; et al. Unusual Thermal Hall Effect in a Kitaev Spin Liquid Candidate $\alpha-\mathrm{RuCl}_{3}$. Phys. Rev. Lett. 2018, 120, 217205. [CrossRef]

39. Affleck, I.; Lieb, E. A Proof of Part of Haldane's Conjecture on Spin Chains. Lett. Math. Phys. 1986, 12, 57-69. [CrossRef]

40. Affleck, I.; Kennedy, T.; Lieb, E.H.; Tasaki, H. Rigorous Results on Valence-Bond Ground States in Antiferromagnets. Phys. Rev. Lett. 1987, 59, 799-802. [CrossRef]

41. Giamarchi, T. Quantum Physics in One Dimension, 1st ed.; Clarendon Press: Oxford, UK, 2003; ISBN 9780198525004.

42. Tennant, D.H.; Cowley, R.A.; Nagler, S.E.; Tsvelik, A.M. Measurement of the Spin-Excitation Continuum in One-Dimensional $\mathrm{KCuF}_{3}$ using Neutron Scattering. Phys. Rev. B 1995, 52, 13368-13380. [CrossRef]

43. Coldea, R.; Tennant, D.A.; Cowley, R.A.; McMorrow, D.F.; Dorner, B.; Tylczynski, Z. Quasi-1D S = 1/2 Antiferromagnet $\mathrm{Cs}_{2} \mathrm{CuCl}_{4}$ in a Magnetic Field. Phys. Rev. Lett. 1997, 79, 151-154. [CrossRef]

44. Stone, M.B.; Reich, D.H.; Broholm, C.; Lefmann, K.; Rischel, C.; Landee, C.P.; Turnbull, M.M. Extended Quantum Critical Phase in a Magnetized Spin-1/2 Antiferromagnetic Chain. Phys. Rev. Lett. 2003, 91, 037205. [CrossRef] [PubMed]

45. Bonner, J.C.; Fisher, M.E. Linear Magnetic Chains with Anisotropic Coupling. Phys. Rev. 1964, 135, A640-A658. [CrossRef]

46. Blöte, H.W.J. The Specific Heat of Magnetic Linear Chains. Physica B \& C 1975, 79, 427-466. [CrossRef]

47. Klümper, A.; Johnston, D.C. Thermodynamics of the Spin-1/2 Antiferromagnetic Uniform Heisenberg Chain. Phys. Rev. Lett. 2000, 84, 4701-4704. [CrossRef] [PubMed] 
48. Johnston, D.C.; Kremer, R.K.; Troyer, M.; Wang, X.; Klümper, A.; Bud'ko, S.L.; Panchula, A.F.; Canfield, P.C. Thermodynamics of Spin $S=1 / 2$ Antiferromagnetic Uniform and Alternating-Exchange Heisenberg Chains. Phys. Rev. B 2000, 61, 9558-9606. [CrossRef]

49. Tarasenko, R.; Čižmár, E.; Orendáčová, A.; Kuchár, J.; Černák, J.; Prokleška, J.; Sechovský, V.; Orendáč, M. $\mathrm{S}=1 / 2$ Heisenberg Antiferromagnetic Spin Chain $\left[\mathrm{Cu}(\mathrm{dmbpy})\left(\mathrm{H}_{2} \mathrm{O}\right)_{2} \mathrm{SO}_{4}\right]\left(\mathrm{dmbpy}=4,4^{\prime}\right.$-dimethyl2,2'-bipyridine): Synthesis, Crystal Structure and Enhanced Magnetocaloric Effect. Solid State Sci. 2014, 28, 14-19. [CrossRef]

50. Piazza, B.D.; Mourigal, M.; Christensen, N.B.; Nilsen, G.J.; Tregenna-Piggott, P.; Perring, T.G.; Enderle, M.; McMorrow, D.F.; Ivanov, D.A.; Ronnow, H.M. Fractional Excitations in the Square-Lattice Quantum Antiferromagnet. Nat. Phys. 2015, 11, 62-68. [CrossRef]

51. Chakravarty, S.; Halperin, B.I.; Nelson, D.R. Low-Temperature Behavior of Two-Dimensional Quantum Antiferromagnets. Phys. Rev. Lett. 1988, 60, 1057-1060. [CrossRef]

52. Chakravarty, S.; Halperin, B.I.; Nelson, D.R. Two-Dimensional Quantum Heisenberg Antiferromagnet at Low Temperatures. Phys. Rev. B 1989, 39, 2344-2371. [CrossRef]

53. Makivić, M.S.; Ding, H.-Q. Two-Dimensional Spin-1/2 Heisenberg Antiferromagnet: A Quantum Monte Carlo Study. Phys. Rev. B 1991, 43, 3562-3574. [CrossRef]

54. Cuccoli, A.; Tognetti, V.; Vaia, R.; Verrucchi, P. Two-Dimensional Quantum Heisenberg Antiferromagnet: Effective-Hamiltonian Approach to the Thermodynamics. Phys. Rev. B 1997, 56, 14456-14468. [CrossRef]

55. Kim, J.-K.; Troyer, M. Low Temperature Behavior and Crossovers of the Square Lattice Quantum Heisenberg Antiferromagnet. Phys. Rev. Lett. 1998, 80, 2705-2708. [CrossRef]

56. Orendáč, M.; Orendáčová, A.; Černák, J.; Feher, A. Magnetic Specific Heat Analysis of $\mathrm{Cu}\left(\mathrm{C}_{2} \mathrm{H}_{8} \mathrm{~N}_{2}\right)_{2} \mathrm{Ni}(\mathrm{CN})_{4}$ : A Quasi-Two-Dimensional Heisenberg Antiferromagnet. Solid State Commun. 1995, 94, 833-835. [CrossRef]

57. Hanko, J.; Orendáč, M.; Kuchár, J.; Žák, Z.; Černák, J.; Orendáčová, A.; Feher, A. Hydrogen Bonds Mediated Magnetism in $\mathrm{Cu}(\mathrm{bmen})_{2} \mathrm{Pd}(\mathrm{CN})_{4}$. Solid State Commun. 2007, 142, 128-131. [CrossRef]

58. Turnbull, M.M.; Albrecht, A.S.; Jameson, G.B.; Landee, C.P. High-Field Magnetization Studies of Two-Dimensional Copper Antiferromagnets. Mol. Cryst. Liq. Cryst. 1999, 335, 245-252. [CrossRef]

59. Xiao, F.; Woodward, F.M.; Landee, C.P.; Turnbull, M.M.; Mielke, C.; Harrison, N.; Lancaster, T.; Blundell, S.J.; Baker, P.J.; Babkevich, P.; et al. Two-Dimensional XY Behavior Observed in Quasi-Two-Dimensional Quantum Heisenberg Antiferromagnets. Phys. Rev. B 2009, 79, 134412. [CrossRef]

60. Clarke, S.J.; Harrison, A.; Mason, T.E.; Visser, D. Characterisation of Spin-Waves in Copper(II) Deuteroformate Tetradeuterate: A Square S = 1/2 Heisenberg Antiferromagnet. Solid State Commun. 1999, 112, 561-564. [CrossRef]

61. Zhou, P.; Drumheller, J.E.; Rubenacker, G.V.; Halvorson, K.; Willett, R.D. Novel Low-Dimensional Spin 1/2 Antiferromagnets: Two-Halide Exchange Pathways in $\mathrm{A}_{2} \mathrm{CuBr}_{4}$ Salts. J. Appl. Phys. 1991, 69, 5804. [CrossRef]

62. Woodward, F.M.; Landee, C.P.; Giantsidis, J.; Turnbull, M.M.; Richardson, C. Structure and Magnetic Properties of $(5 \mathrm{BAP})_{2} \mathrm{CuBr}_{4}$ : Magneto-Structural Correlations of Layered $\mathrm{S}=1 / 2$ Heisenberg Antiferromagnets. Inorg. Chim. Acta 2001, 324, 324-330. [CrossRef]

63. Woodward, F.M.; Albrecht, A.S.; Wynn, C.M.; Landee, C.P.; Turnbull, M.M. Two-Dimensional S = 1/2 Heisenberg Antiferromagnets: Synthesis, Structure, and Magnetic Properties. Phys. Rev. B 2002, 65, 144412. [CrossRef]

64. Coomer, F.C.; Bondah-Jagalu, V.; Grant, K.J.; Harrison, A.; McIntyre, G.J.; Rønnow, H.M.; Feyerherm, R.; Wand, T.; Meißner, M.; Visser, D.; et al. Neutron Diffraction Studies of Nuclear and Magnetic Structures in the $\mathrm{S}=1 / 2$ Square Heisenberg Antiferromagnets $\left(\mathrm{d}_{6}-5 \mathrm{CAP}\right)_{2} \mathrm{CuX}_{4}(\mathrm{X}=\mathrm{Br}$ and $\mathrm{Cl})$. Phys. Rev. B 2007, 75, 094424. [CrossRef]

65. Kajňaková, M.; Orendáč, M.; Orendáčová, A.; Vlček, A.; Černák, J.; Kravchyna, O.V.; Anders, A.G.; Bałanda, M.; Park, J.-H.; Feher, A.; et al. $\mathrm{Cu}\left(\mathrm{H}_{2} \mathrm{O}\right)_{2}\left(\mathrm{C}_{2} \mathrm{H}_{8} \mathrm{~N}_{2}\right) \mathrm{SO}_{4}$ : A Quasi-Two-Dimensional S = 1/2 Heisenberg Antiferromagnet. Phys. Rev. B 2005, 71, 014435. [CrossRef]

66. Potočňák, I.; Vavra, M.; Čižmár, E.; Tibenská, K.; Orendáčová, A.; Steinbornc, D.; Wagner, C.; Dušek, M.; Fejfarová, K.; Schmidt, H.; et al. Low-Dimensional Compounds Containing Cyano Groups. XIV. Crystal Structure, Spectroscopic, Thermal and Magnetic Properties of $\left[\mathrm{Cu} L_{2}\right]\left[\mathrm{Pt}(\mathrm{CN})_{4}\right]$ Complexes ( $L=$ ethylenediamine or $N, N$-dimethylethylenediamine). J. Solid State Chem. 2006, 179, 1965-1976. [CrossRef] 
67. Zeleňák, V.; Orendáčová, A.; Císařová, I.; Černák, J.; Kravchyna, O.V.; Park, J.-H.; Orendáč, M.; Anders, A.G.; Feher, A.; Meisel, M.W. Magneto-Structural Correlations in $\mathrm{Cu}(\mathrm{tn}) \mathrm{Cl}_{2}$ (tn = 1,3-Diaminopropane): Two-Dimensional Spatially Anisotropic Triangular Magnet Formed by Hydrogen Bonds. Inorg. Chem. 2006, 45, 1774-1782. [CrossRef] [PubMed]

68. Vlček, A.; Orendáč, M.; Orendáčová, A.; Kajňaková, M.; Papageorgiou, T.; Chomič, J.; Černák, J.; Massa, W.; Feher, A. Magneto-Structural Correlation in $\mathrm{Cu}\left(\mathrm{NH}_{3}\right)_{2} \mathrm{Ag}_{2}(\mathrm{CN})_{4}$. Crystal Structure, Magnetic and Thermodynamic Properties of an $S=1 / 2$ Low-Dimensional Heisenberg Antiferromagnet. Solid State Sci. 2007, 9, 116-125. [CrossRef]

69. Sedláková, L.; Tarasenko, R.; Potočňák, I.; Orendáčová, A.; Kajňaková, M.; Orendáč, M.; Starodub, V.A.; Anders, A.G.; Kravchyna, O.; Feher, A. Magnetic Properties of $S=1 / 2$ Two-Dimensional Quantum Antiferromagnet $\mathrm{Cu}\left(\mathrm{D}_{2} \mathrm{O}\right)_{2}\left(\mathrm{C}_{2} \mathrm{H}_{6} \mathrm{D}_{2} \mathrm{~N}_{2}\right) \mathrm{SO}_{4}$. Solid State Commun. 2008, 147, 239-241. [CrossRef]

70. Orendáč, M.; Čižmár, E.; Orendáčová, A.; Tkáčová, J.; Kuchár, J.; Černák, J. Enhanced Magnetocaloric Effect in Quasi-One-Dimensional $S=1 / 2$ Heisenberg Antiferromagnet $\left[\mathrm{Cu}(\mathrm{dmen})_{2}\left(\mathrm{H}_{2} \mathrm{O}\right)\right] \mathrm{SiF}_{6}$. J. Alloys Compd. 2014, 586, 34-38. [CrossRef]

71. Parola, A.; Sorella, S.; Zhong, Q.F. Realization of a Spin Liquid in a Two Dimensional Quantum Antiferromagnet. Phys. Rev. Lett. 1993, 71, 4393-4396. [CrossRef]

72. Ihle, D.; Schindelin, C.; Weiße, A.; Fehske, H. Magnetic Order-Disorder Transition in the Two-Dimensional Spatially Anisotropic Heisenberg Model at Zero Temperature. Phys. Rev. B 1999, 60, 9240-9243. [CrossRef]

73. Affleck, I.; Halperin, B. On a Renormalization Group Approach to Dimensional Crossover. J. Phys. A Math. Gen. 1996, 29, 2627-2631. [CrossRef]

74. Sandvik, A.W. Multichain Mean-Field Theory of Quasi-One-Dimensional Quantum Spin Systems. Phys. Rev. Lett. 1999, 83, 3069-3072. [CrossRef]

75. Kim, Y.J.; Birgeneau, R.J. Monte Carlo Study of the $S=1 / 2$ and $S=1$ Heisenberg Antiferromagnet on a Spatially Anisotropic Square Lattice. Phys. Rev. B 2000, 62, 6378-6384. [CrossRef]

76. Jiang, F.-J.; Kämpfer, F.; Nyfeler, M. Monte Carlo Determination of the Low-Energy Constants of a Spin-1/2 Heisenberg Model with Spatial Anisotropy. Phys. Rev. B 2009, 80, 033104. [CrossRef]

77. Keith, B.C.; Landee, C.P.; Valleau, T.; Turnbull, M.M.; Harrison, N. Two-Dimensional Spin-1/2 Rectangular Heisenberg antiferromagnets: Simulation and Experiment. Phys. Rev. B 2011, 84, 104442; Erratum Phys. Rev. B 2011, 84, 229901, doi:10.1103/PhysRevB.84.229901. [CrossRef]

78. Cliffe, M.J.; Lee, J.; Paddison, J.A.M.; Schott, S.; Mukherjee, P.; Gaultois, M.W.; Manuel, P.; Sirringhaus, H.; Dutton, S.E.; Grey, C.P. Low-Dimensional Quantum Magnetism in Cu(NCS) 2 : A Molecular Framework Material. Phys. Rev. B 2018, 97, 144421. [CrossRef]

79. Nath, R.; Padmanabhan, M.; Baby, S.; Thirumurugan, A.; Ehlers, D.; Hemmida, M.; Krug von Nidda, H.-A.; Tsirlin, A.A. Quasi-Two-Dimensional $\mathrm{S}=1 / 2$ Magnetism of $\mathrm{Cu}\left[\mathrm{C}_{6} \mathrm{H}_{2}(\mathrm{COO})_{4}\right]\left[\mathrm{C}_{2} \mathrm{H}_{5} \mathrm{NH}_{3}\right]_{2}$. Phys. Rev. B 2015, 91, 054409. [CrossRef]

80. Tarasenko, R.; Orendáčová, A.; Čižmár, E.; Mat’aš, S.; Orendáč, M.; Potočňák, I.; Siemensmeyer, K.; Zvyagin, S.; Wosnitza, J.; Feher, A. Spin Anisotropy in $\mathrm{Cu}(\mathrm{en})\left(\mathrm{H}_{2} \mathrm{O}\right)_{2} \mathrm{SO}_{4}$ : A Quasi-Two-Dimensional $\mathrm{S}=1 / 2$ Spatially Anisotropic Triangular-Lattice Antiferromagnet. Phys. Rev. B 2013, 87, 174401. [CrossRef]

81. Sýkora, R.; Legut, D. Magnetic Interactions in a Quasi-One-Dimensional Antiferromagnet $\mathrm{Cu}\left(\mathrm{H}_{2} \mathrm{O}\right)_{2}(\mathrm{en}) \mathrm{SO}_{4}$. J. Appl. Phys. 2014, 115, 17B305. [CrossRef]

82. Lederová, L.; Orendáčová, A.; Chovan, J.; Strečka, J.; Verkholyak, T.; Tarasenko, R.; Legut, D.; Sýkora, R.; Čižmár, E.; Tkáč, V.; et al. Realization of a Spin-1/2 Spatially Anisotropic Square Lattice in a Quasi-Two-Dimensional Quantum Antiferromagnet $\mathrm{Cu}(\mathrm{en})\left(\mathrm{H}_{2} \mathrm{O}\right)_{2} \mathrm{SO}_{4}$. Phys. Rev. B 2017, 95, 054436. [CrossRef]

83. Barbero, N.; Shiroka, T.; Landee, C.P.; Pikulski, M.; Ott, H.-R.; Mesot, J. Pressure and Magnetic Field Effects on a Quasi-Two-Dimensional Spin-1/2 Heisenberg Antiferromagnet. Phys. Rev. B 2016, 93, 054425. [CrossRef]

84. Goddard, P.A.; Singleton, J.; Franke, I.; Möller, J.S.; Lancaster, T.; Steele, A.J.; Topping, C.V.; Blundell, S.J.; Pratt, F.L.; Baines, C.; et al. Control of the Third Dimension in Copper-Based Square-Lattice Antiferromagnets. Phys. Rev. B 2016, 93, 094430. [CrossRef]

85. Yamaguchi, H.; Tamekuni, Y.; Iwasaki, Y.; Otsuka, R.; Hosokoshi, Y.; Kida, T.; Hagiwara, M. Magnetic Properties of a Quasi-Two-Dimensional $S=1 / 2$ Heisenberg Antiferromagnet with Distorted Square Lattice. Phys. Rev. B 2017, 95, 235135. [CrossRef] 
86. Siurakshina, L.; Ihle, D.; Hayn, R. Theory of Magnetic Order in the Three-Dimensional Spatially Anisotropic Heisenberg Model. Phys. Rev. B 2000, 61, 14601-14606. [CrossRef]

87. Sengupta, P.; Sandvik, A.W.; Singh, R.R.P. Specific Heat of Quasi-Two-Dimensional Antiferromagnetic Heisenberg Models with Varying Interplanar Couplings. Phys. Rev. B 2003, 68, 094423. [CrossRef]

88. Yasuda, C.; Todo, S.; Hukushima, K.; Alet, F.; Keller, M.; Troyer, M.; Takayama, H. Néel Temperature of Quasi-Low-Dimensional Heisenberg Antiferromagnets. Phys. Rev. Lett. 2005, 94, 217201. [CrossRef] [PubMed]

89. Orendáčová, A.; Čižmár, E.; Sedláková, L.; Hanko, J.; Kajňaková, M.; Orendáč, M.; Feher, A.; Xia, J.S.; Yin, L.; Pajerowski, D.M.; et al. Interplay of Frustration and Magnetic Field in the Two-Dimensional Quantum Antiferromagnet $\mathrm{Cu}(\mathrm{tn}) \mathrm{Cl}_{2}$. Phys. Rev. B 2009, 80, 144418. [CrossRef]

90. Kohama, Y.; Jaime, M.; Ayala-Valenzuela, O.E.; McDonald, R.D.; Mun, E.D.; Corbey, J.F.; Manson, J.L. Field-Induced XY and Ising Ground States in a Quasi-Two-Dimensional S = 1/2 Heisenberg Antiferromagnet. Phys. Rev. B 2011, 84, 184402. [CrossRef]

91. Irkhin, V.Y.; Katanin, A.A. Thermodynamics of Isotropic and Anisotropic Layered Magnets: Renormalization-Group Approach and 1/N Expansion. Phys. Rev. B 1998, 57, 379-391. [CrossRef]

92. Hathaway, B.J.; Billing, D.E. The Electronic Properties and Stereochemistry of Mono-Nuclear Complexes of the Copper(II) Ion. Coord. Chem. Rev. 1970, 5, 143-207. [CrossRef]

93. Moriya, T. Anisotropic Superexchange Interaction and Weak Ferromagnetism. Phys. Rev. 1960, 120, 91-98. [CrossRef]

94. Povarov, K.Y.; Smirnov, A.I.; Landee, C.P. Switching of Anisotropy and Phase Diagram of the Heisenberg Square-Lattice $\mathrm{S}=1 / 2$ Antiferromagnet $\mathrm{Cu}(\mathrm{pz})_{2}\left(\mathrm{ClO}_{4}\right)_{2}$. Phys. Rev. B 2013, 87, 214402. [CrossRef]

95. Ihle, D.; Schindelin, C.; Fehske, H. Magnetic Order in the Quasi-Two-Dimensional Easy-Plane XXZ Model. Phys. Rev. B 2001, 64, 054419. [CrossRef]

96. Cuccoli, A.; Roscilde, T.; Vaia, R.; Verrucchi, P. Detection of XY Behavior in Weakly Anisotropic Quantum Antiferromagnets on the Square Lattice. Phys. Rev. Lett. 2003, 90, 167205. [CrossRef] [PubMed]

97. Cuccoli, A.; Roscilde, T.; Tognetti, V.; Vaia, R.; Verrucchi, P. Quantum Monte Carlo Study of S =1/2 Weakly Anisotropic Antiferromagnets on the Square Lattice. Phys. Rev. B 2003, 67, 104414. [CrossRef]

98. Pereira, A.R.; Pires, A.S.T. Dynamics of Vortices in a Two-Dimensional Easy-Plane Antiferromagnet. Phys. Rev. B 1995, 51, 996-1002. [CrossRef]

99. Cuccoli, A.; Gori, G.; Vaia, R.; Verrucchi, P. Phase Diagram of the Two-Dimensional Quantum Antiferromagnet in a Magnetic Field. J. Appl. Phys. 2006, 99, 08H503. [CrossRef]

100. de Groot, H.J.M.; de Jongh, L.J. Phase Diagrams of Weakly Anisotropic Heisenberg Antiferromagnets, Nonlinear Excitations (Solitons) and Random-Field Effects. Physica B \& C 1986, 141, 1-36. [CrossRef]

101. Landau, D.P.; Binder, K. Phase Diagrams and Critical Behavior of a Two-Dimensional Anisotropic Heisenberg Antiferromagnet. Phys. Rev. B 1981, 24, 1391-1403. [CrossRef]

102. Holtschneider, M.; Selke, W.; Leidl, R. Two-Dimensional Anisotropic Heisenberg Antiferromagnet in a Magnetic Field. Phys. Rev. B 2005, 72, 064443. [CrossRef]

103. Zhou, C.; Landau, D.P.; Schulthess, T.C. Hidden Zero-Temperature Bicritical Point in the Two-Dimensional Anisotropic Heisenberg Model: Monte Carlo Simulations and Proper Finite-Size Scaling. Phys. Rev. B 2006, 74, 064407. [CrossRef]

104. Pelissetto, A.; Vicari, E. Multicritical Behavior of Two-Dimensional Anisotropic Antiferromagnets in a Magnetic Field. Phys. Rev. B 2007, 76, 024436. [CrossRef]

105. Pires, A.S.T. Kosterlitz-Thouless Transition in a Two-Dimensional Isotropic Antiferromagnet in a Uniform Field. Phys. Rev. B 1994, 50, 9592-9594. [CrossRef]

106. Cuccoli, A.; Roscilde, T.; Vaia, R.; Verrucchi, P. Field-Induced XY Behavior in the $S=1 / 2$ Antiferromagnet on the Square Lattice. Phys. Rev. B 2003, 68, 060402. [CrossRef]

107. Cuccoli, A.; Roscilde, T.; Vaia, R.; Verrucchi, P. XY Behaviour of the 2D Antiferromagnet in a Field. J. Magn. Magn. Mater. 2004, 884, 272-276. [CrossRef]

108. Sengupta, P.; Batista, C.D.; McDonald, R.D.; Cox, S.; Singleton, J.; Huang, L.; Papageorgiou, T.P.; Ignatchik, O.; Herrmannsdörfer, T.; Manson, J.L.; et al. Nonmonotonic Field Dependence of the Néel Temperature in the Quasi-Two-Dimensional Magnet $\left[\mathrm{Cu}\left(\mathrm{HF}_{2}\right)\left(\mathrm{pyz}_{2}\right] \mathrm{BF}_{4}\right.$. Phys. Rev. B 2009, 79, 060409. [CrossRef] 
109. Tsyrulin, N.; Xiao, F.; Schneidewind, A.; Link, P.; Rønnow, H.M.; Gavilano, J.; Landee, C.P.; Turnbull, M.M.; Kenzelmann, M. Two-Dimensional Square-Lattice $\mathrm{S}=1 / 2$ Antiferromagnet $\mathrm{Cu}(\mathrm{pz})_{2}\left(\mathrm{ClO}_{4}\right)_{2}$. Phys. Rev. B 2010, 81, 134409. [CrossRef]

110. Fortune, N.A.; Hannahs, S.T.; Landee, C.P.; Turnbull, M.M.; Xiao, F. Magnetic-Field-Induced Heisenberg to XY Crossover in a Quasi-2D Quantum Antiferromagnet. J. Phys. Conf. Ser. 2014, 568, 042004. [CrossRef]

111. Glazkov, V.N.; Krasnikova, Y.; Rodygina, I.; Tarasenko, R.; Orendáčová, A. Low-Temperature Antiferromagnetic Resonance of Quasi-2D Magnet $\mathrm{Cu}(\mathrm{en})\left(\mathrm{H}_{2} \mathrm{O}\right)_{2} \mathrm{SO}_{4}$. In Proceedings of the International Symposium Spin Waves 2018, St. Petersburg, Russia, 3-8 June 2018.

112. Kubo, Y.; Kurihara, S. Tunable Rotons in Square-Lattice Antiferromagnets under Strong Magnetic Fields. Phys. Rev. B 2014, 90, 014421. [CrossRef]

113. Powalski, M.; Uhrig, G.S.; Schmidt, K.P. Roton Minimum as a Fingerprint of Magnon-Higgs Scattering in Ordered Quantum Antiferromagnets. Phys. Rev. Lett. 2015, 115, 207202. [CrossRef]

114. Orendáčová, A.; Kajňaková, M.; Černák, J.; Park, J.-H.; Čižmár, E.; Orendáč, M.; Vlček, A.; Kravchyna, O.V.; Anders, A.G.; Feher, A.; et al. Hydrogen Bond Mediated Magnetism in $\left[\mathrm{Cu}^{\mathrm{II}}(\mathrm{en})_{2}\left(\mathrm{H}_{2} \mathrm{O}\right)\right]$ $\left[\mathrm{Cu}^{\mathrm{II}}(\mathrm{en})_{2} \mathrm{Ni}_{2} \mathrm{Cu}_{2}{ }_{2}(\mathrm{CN})_{10}\right] \cdot 2 \mathrm{H}_{2} \mathrm{O}$. Chem. Phys. 2005, 309, 115-125. [CrossRef]

115. Brown, S.; Cao, J.; Musfeldt, J.L.; Conner, M.M.; McConnel, A.C.; Southerland, H.I.; Manson, J.L.; Schlueter, J.A.; Phillips, M.D.; Turnbull, M.M.; et al. Hydrogen Bonding and Multiphonon Structure in Copper Pyrazine Coordination Polymers. Inorg. Chem. 2007, 46, 8577-8583. [CrossRef] [PubMed]

116. Legut, D.; Sýkora, R.; Wdowik, U.D.; Orendáčová, A. Mechanical Properties of the Quasi-One-Dimensional Antiferromagnet $\mathrm{Cu}(\mathrm{en})\left(\mathrm{H}_{2} \mathrm{O}\right)_{2} \mathrm{SO}_{4}$. J. Nanosci. Nanotechnol. 2018, 18, 3016-3018. [CrossRef] [PubMed]

117. Perren, G.; Möller, J.S.; Hüvonen, D.; Podlesnyak, A.A.; Zheludev, A. Spin Dynamics in Pressure-Induced Magnetically Ordered Phases in $\left(\mathrm{C}_{4} \mathrm{H}_{12} \mathrm{~N}_{2}\right) \mathrm{Cu}_{2} \mathrm{Cl}_{6}$. Phys. Rev. B 2015, 92, 054413. [CrossRef]

118. O’Neal, K.R.; Zhou, J.; Cherian, J.G.; Turnbull, M.M.; Landee, C.P.; Jena, P.; Liu, Z.; Musfeldt, J.L. Pressure-Induced Structural Transition in Copper Pyrazine Dinitrate and Implications for Quantum Magnetism. Phys. Rev. B 2016, 93, 104409. [CrossRef]

119. Cini, A.; Mannini, M.; Totti, F.; Fittipaldi, M.; Spina, G.; Chumakov, A.; Rüffer, R.; Cornia, A.; Sessoli, R. Mössbauer Spectroscopy of a Monolayer of Single Molecule Magnets. Nat. Commun. 2018, 9, 480. [CrossRef]

120. Campbell, V.E.; Tonelli, M.; Cimatti, I.; Moussy, J.-B.; Tortech, L.; Dappe, Y.J.; Rivie, E.; Guillot, R.; Delprat, S.; Mattana, R.; et al. Engineering the Magnetic Coupling and Anisotropy at the Molecule-Magnetic Surface Interface in Molecular Spintronic Devices. Nat. Commun. 2016, 7, 13646. [CrossRef]

121. Laskowska, M.; Kityk, I.; Dulski, M.; Jędryka, J.; Wojciechowski, A.; Jelonkiewicz, J.; Wojtyniak, M.; Laskowski, Ł. Functionalized Mesoporous Silica Thin Films as a Tunable Nonlinear Optical Material. Nanoscale 2017, 9, 12110-12123. [CrossRef]

(C) 2018 by the authors. Licensee MDPI, Basel, Switzerland. This article is an open access article distributed under the terms and conditions of the Creative Commons Attribution (CC BY) license (http://creativecommons.org/licenses/by/4.0/). 